\title{
Advances in the Modelling of Motorcycle Dynamics
}

\author{
R.S. SHARP, S. EVANGELOU and D.J.N. LIMEBEER \\ Electrical and Electronic Engineering, Imperial College London, South Kensington Campus, \\ London SW7 2AZ, UK; E-mail: robin.sharp@imperial.ac.uk
}

(Received: 7 July 2003; accepted in revised form: 7 June 2004)

\begin{abstract}
Starting from an existing advanced motorcycle dynamics model, which allows simulation of reasonably general motions and stability, modal and response computations for small perturbations from any trim condition, improvements are described. These concern (a) tyre/road contact geometry, (b) tyre shear force and moment descriptions, as functions of load, slip and camber, (c) tyre relaxation properties, (d) a new analytic treatment of the monoshock rear suspension mechanism with sample results, (e) parameter values describing a contemporary high performance machine and rider, (f) steady-state equilibrium and power checking and (g) steering control. In particular, the "Magic Formula" motorcycle tyre model is utilised and complete sets of parameter values for contemporary tyres are derived by identification methods. The new model is used for steady turning, stability, design parameter sensitivity and response to road forcing calculations. The results show the predictions of the model to be in general agreement with observations of motorcycle behaviour from the field and they suggest that frame flexibility remains an important design and analysis area, despite improvements in frame designs over recent years. Motorcycle rider parameters have significant influences on the behaviour, with results consistent with a commonly held view, that lightweight riders are more likely to suffer oscillation problems than heavyweight ones.
\end{abstract}

Keywords: motorcycle, tyre, contact, monoshock, stability, response, sensitivity.

\section{Introduction}

The handling qualities of motorcycles are often of great importance. They affect the pleasure to be gained from the rider-machine interactions and the safety of the rider. Self-steering action is crucial with single track vehicles and rider control is primarily by steering torque, so-called free-control [1]. A consequence of the free steering system is that motorcycles are oscillatory. Several modes of motion potentially have small damping factors. Therefore much attention must be directed towards controlling the oscillatory tendencies, throughout the operating range. Also, it is desirable that motorcycles are responsive to the rider's commands and stability should not be pursued without reference to other qualities.

In straight running, motorcycles are substantially symmetric and in-plane and out-of-plane motions are decoupled at first order level $[1,2]$. In cornering, inplane and out-of-plane cross-coupling makes any effective analysis of the dynamics complicated. Automated multibody dynamics analysis software [3-7] has opened up the topic significantly in recent years. The steady turning problem can be solved, 
possibly with the aid of a stabilising steering controller, and modal analysis can be completed for small perturbations from any equilibrium "trim" state.

Accuracy of predicted behaviour depends, not only on effective conceptual modelling and multibody analysis, but also on good parameter values. Central issues in modelling include the representations of frame flexibilities, tyre-road contact geometry and tyre shear forces. Many previous findings relate to motorcycle and tyre descriptions which are now somewhat dated and to tyre models which have a limited domain of applicability. It is therefore of interest (i) to obtain a parametric description of a modern machine, (ii) to utilise a more comprehensive tyre force model, with parameter values to correspond to a modern set of tyres, (iii) to determine steady turning, stability, response and parameter sensitivity data for comparison with older information, to determine to what extent it remains valid, and (iv) to better understand the design of modern machines. The paper is subsequently an account of such work. Novel analysis of a "monoshock" rear suspension system is also included.

\section{Parametric Description of a Modern Motorcycle}

The authors are currently engaged in a measurement campaign to obtain the relevant parameters of a Suzuki GSX-R1000K1 machine. Such a motorcycle has been disassembled and many of its parts have been measured, starting with the lighter ones. At this stage, the campaign is incomplete. In particular, the frame stiffness and damping parameters used and the location of the elastic centre are currently only estimates.

\subsection{GEOMETRY AND MASSES}

The workshop manual for the motorcycle includes pictures to scale and key dimensions, like the wheelbase and the steering head angle. Joints between components at the steering head and the swing arm pivot can be identified there and many key points, including those related to the monoshock rear suspension, can be located with reasonable precision from these pictures. A scaled diagrammatic representation of the motorcycle is shown in Figure 1, the corresponding parameter values being included in an Appendix. The front frame has been measured separately to give the points $\mathrm{p} 3$ and $\mathrm{p} 5$. The point $\mathrm{p} 4$ is along the line of the lower front fork translation relative to the upper forks. The estimated location $\mathrm{p} 2$ is the elastic centre of the rear frame with respect to a moment perpendicular to the steer axis.

The rider's total mass is taken as $72 \mathrm{~kg}, 62 \%$ of which is associated with the upper body. The masses of the hands and half of the lower arms may be considered to be part of the steering system. The rider parameters derive from bio-mechanical data [8], accounting for his posture on the machine.

Circles representing the body mass centres are in proportion to the masses concerned, which are known through straightforward weighing. 


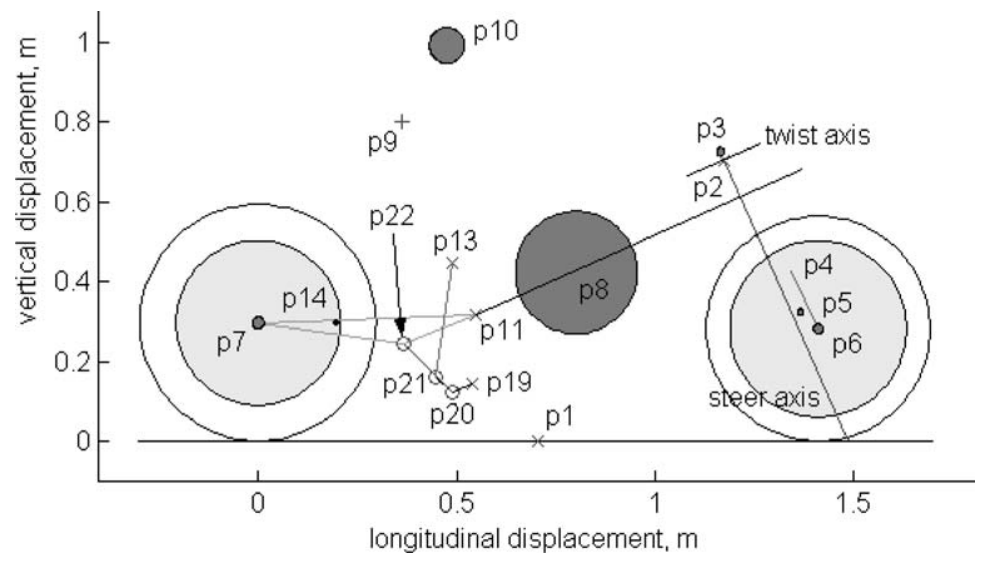

Figure 1. Scaled diagrammatic motorcycle in side view.

\subsection{INERTIAS AND MASS CENTRES}

Wheel and tyre inertias have been obtained by timing oscillations of bi-filar and tri-filar suspension arrangements, utilising axial symmetry in each set-up. Similar bi-filar suspension systems have been used separately for the front and rear frames (Figure 2). Each of these is assumed to have a plane of symmetry and it is clear that the front frame principal axes, in the plane of symmetry, are along and perpendicular to the line of the forks. Oscillation periods, geometric dimensions and the mass of the suspended body lead simply to the moment of inertia about the rotation axis and standard transformations allow the determination of principal inertias and axes for the more complex rear frame [9].

Recent measurements on a driving simulator [10] provide estimates of the contributions to the front frame inertia, steering stiffness and steering damping that arise from the rider's arms and hands, corresponding to relaxed and tense riding. These can be added to the measured values if it is considered appropriate [11]. The swing arm inertias are small enough to be obtained by estimation based on the mass centre location and the dimensions. The wheels have their mass centres at their geometric centres. Other mass centre locations were found using plumb lines and taking photographs (Figure 2). Relevant values are given in the appendix.

\subsection{STIFFNESS AND DAMPING PROPERTIES}

Springs and dampers were tested in a standard dynamic materials testing machine [12]. The maximum actuator velocity available was about $0.25 \mathrm{~m} / \mathrm{s}$, which constrained the damper characteristic measurements. Uni-directional forcing of the steering damper up to the maximum rate of the actuator yielded a substantially linear force/velocity relationship with slope $4340 \mathrm{~N} /(\mathrm{m} / \mathrm{s})$. Using the effective 


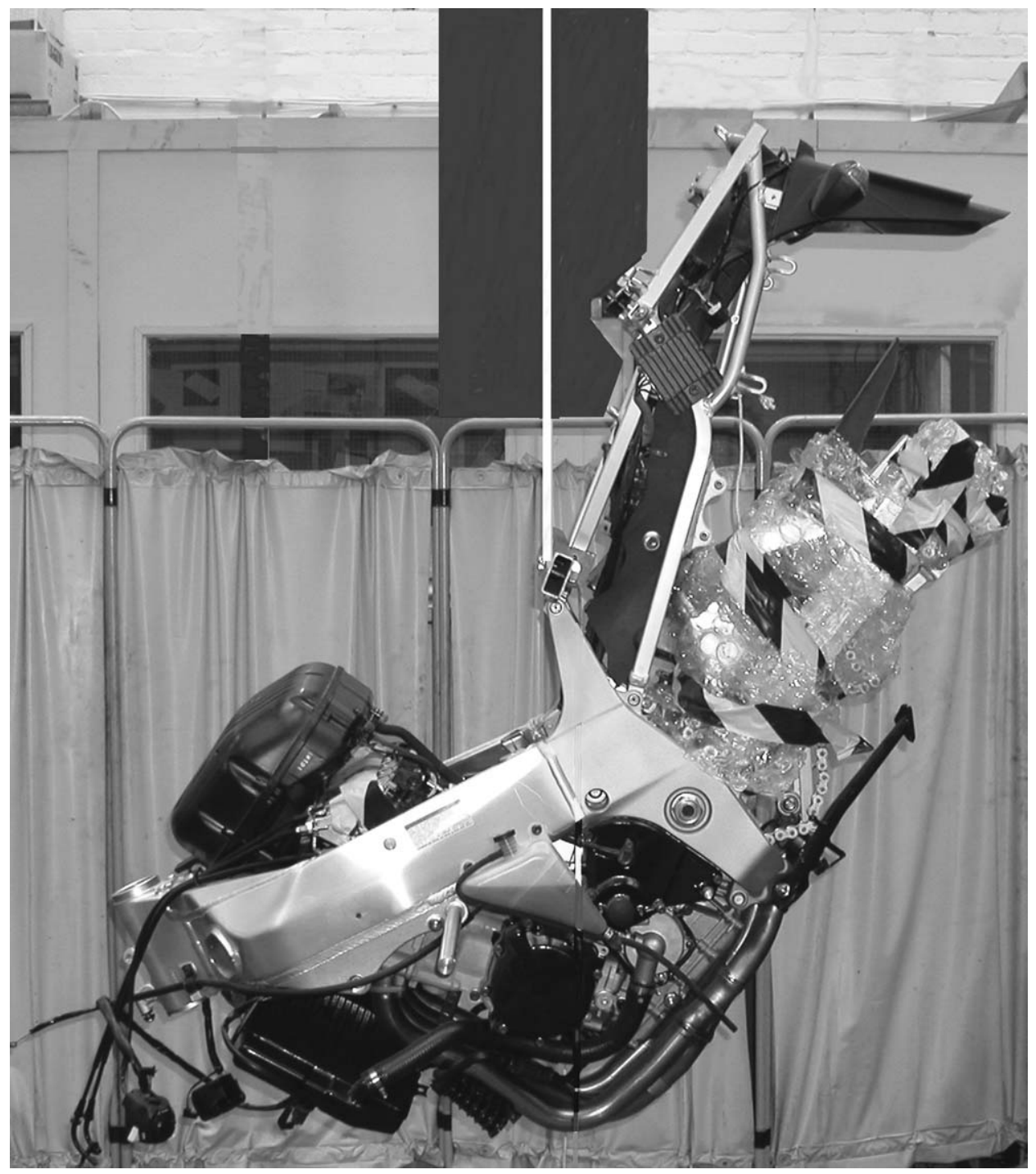

Figure 2. Bifilar suspended motorcycle rear frame for inertia measurement.

moment arm of the damper $(0.04 \mathrm{~m})$ to convert this value to an equivalent rotational coefficient gives a value, $6.944 \mathrm{Nm} /(\mathrm{rad} / \mathrm{s})$.

The dimensions of the single rear steel spring, from the monoshock suspension were measured and the standard helical spring formula, $k=G d^{4} /\left(64 R^{3} n\right)$, was applied to calculating the rate, $k$, as $55 \mathrm{kN} / \mathrm{m}$. The gas filled damper contributes some suspension preload and a small rate, determined from the test machine via static measurements as $3.57 \mathrm{kN} / \mathrm{m}$. The damper unit was stroked at full actuator 
performance first in compression and then in extension, achieving velocities up to about $0.13 \mathrm{~m} / \mathrm{s}$. Allowing for the gas pressure forces in the processing, the damping coefficient in compression was $9.6 \mathrm{kN} /(\mathrm{m} / \mathrm{s})$ and in rebound $13.7 \mathrm{kN} /(\mathrm{m} / \mathrm{s})$. Front spring and damper coefficients are estimates, at this stage. Suspension limit stops are included at each end, modelled as fifth powers of displacement from stop contact. The relevant displacements are known from examination of the parts and from information given in the workshop manual.

The torsional stiffness of the main frame, between the steering head and the power unit, remains to be measured. It is clear from the structural design and materials used that the frame is considerably stiffer than was the norm for tubular framed motorcycles of some years ago. In those cases, it was established that the frame flexibility was an essential contributor to the stability of the wobble mode, in particular $[13,14]$. It remains to be seen how significant this area is for modern machines. The torsional stiffness assumed, at $10^{5} \mathrm{Nm} / \mathrm{rad}$, is 3.5 times that measured statically for a Yamaha 650S [15] and 2.9 times that measured at about the same time by Koenen [2]. Tyre radial stiffnesses come directly from [7].

The rider's upper body has roll freedom relative to the main frame, while the lower body is part of the main frame. The upper body is restrained by a parallel spring damper system. Stiffness and damping parameters are chosen in alignment with the experimental results of Nishimi et al [16], obtained by identifying "rider" parameters in forced vibration on a mock motorcycle frame. The decoupled natural frequency of the rider upper body in roll is $1.27 \mathrm{~Hz}$ and the corresponding damping factor is 0.489 . According to this model, rider resonance will not be apparent due to the high damping factor and it will not be tuned to the machine oscillations, where these are at all vigorous.

\subsection{AERODYNAMICS}

Aerodynamic drag, lift and pitching moment data come from a Triumph motorcycle of similar style and dimensions to the GSX-R1000 [1]. This is steady-state drag force, lift force and pitching moment data from full scale wind tunnel testing, with a prone rider.

\section{Tyre-Road Contact Modelling}

The geometry of the contact between the front tyre and the ground is a relatively complex part of the motorcycle modelling. It is also important to the behaviour of the machine. It has been common to represent the tyre as a thin disc, with the contact point migrating circumferentially for larger camber and steer angles, but Cossalter et al have pioneered the inclusion of tyre width in their descriptions [7, 17-19]. If a disc model is used, it needs to be augmented with an overturning moment description $[2,5]$. This is not necessary with a thick tyre model, since the lateral migration of the contact point then occurs automatically and the overturning moment is a consequence of that movement. A wide tyre with a circular cross-section 


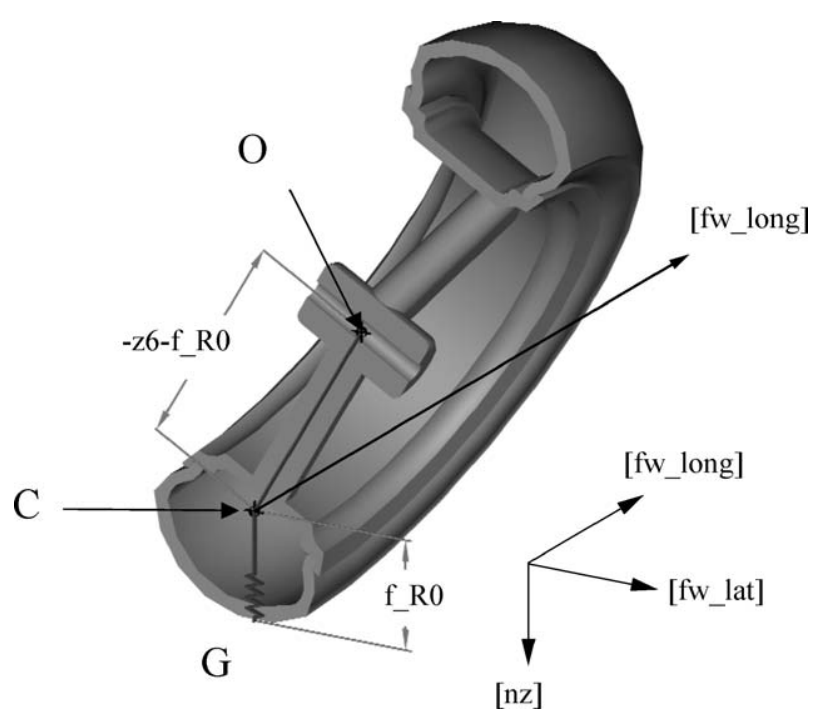

Figure 3. Diagrammatic three-dimensional front wheel contact geometry.

crown is now modelled. In addition to making the overturning moment automatic, longitudinal forces applied to the cambered tyre will lead to realistic aligning moments appearing automatically. A necessary test for the wide tyre model is that it gives the same results as the thin tyre model, when physically equivalent systems are being represented. This test has been applied, with some significant consequences.

To define each tyre/ground contact point (Figures 3 and 4) the vertical and the wheel spindle directions are used in a vector (cross) product to describe the longitudinal direction, with respect to the wheel. Similarly, the wheel radial direction, $O C$ in Figure 3, comes from combining the longitudinal and wheel spindle directions. The vector $O C$ is of fixed length and so is completely specified. $\mathrm{G}$ is vertically below $\mathrm{C}$ and the difference between the tyre crown radius and the distance $C G$ defines the change in the tyre carcass compression from the nominal state and hence the change of the wheel load from the nominal, via the tyre radial stiffness. If the road is profiled, the road height is accounted for in working out the wheel load. The vector $O G=O C+C G$ defines the contact point, which belongs to the wheel but moves within it. $\mathrm{G}$ remains at road surface height but the tyre load cannot become negative. If the tyre leaves the ground, the shear forces are zero, whatever the other conditions are. Tyre forces are applied to the point $\mathrm{G}$, in each case.

The longitudinal slip is the rearward component of the material contact point velocity divided by the absolute value of the rolling velocity, the latter being the forward velocity of the contact point (or the crown centre point, since these are the same). The contact point is defined by its coordinates in the parent body of the wheel and it is de-spun relative to the material contact point. Thus the longitudinal 


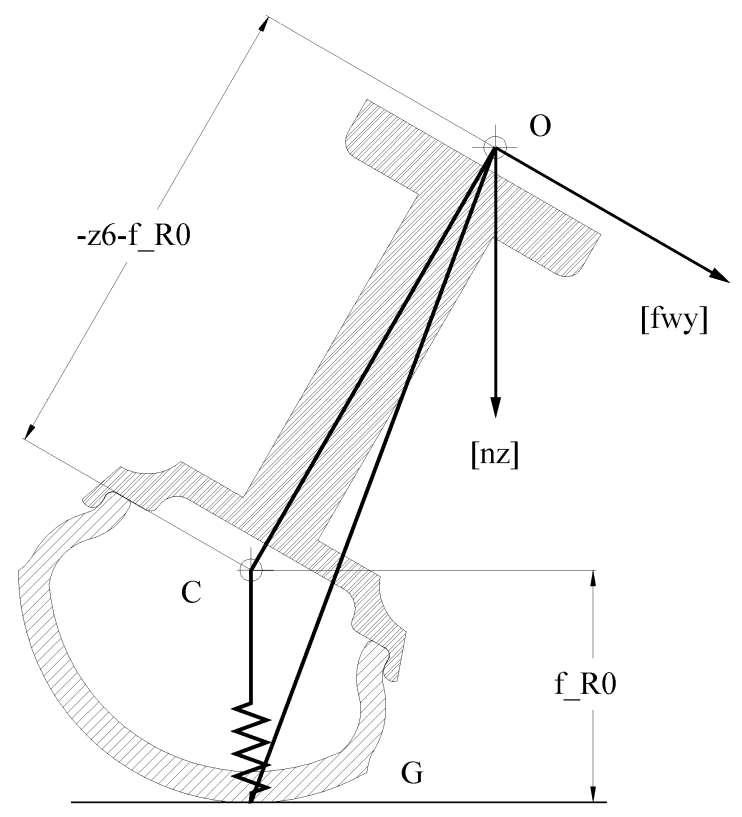

Figure 4. Diagrammatic two-dimensional front wheel contact geometry.

slip is given by an expression of the form:

$$
\begin{aligned}
\kappa= & -(\text { rolling velocity }+ \text { spin component of longitudinal velocity) } \\
& / \text { abs(rolling velocity })
\end{aligned}
$$

The slip angle is the arctangent of the ratio of the (negative) lateral velocity of the tyre contact centre point to the absolute value of the rolling velocity.

In developing this new model from the former one [5], in which the wheels were represented as thin discs, subtle differences between the root locus predictions of the old and new versions were observed in circumstances which were at that stage thought physically equivalent. Such differences were found to be associated with the former description of the slip angles as deriving from the lateral velocity components of the disc tyre contact points. When the wheel camber angle is changing, these points have a small lateral velocity component not connected with sideslipping, since with the real tyre, the contact point moves around the circular section sidewall of the tyre. The former model would have provided a more accurate description if it had used the crown centre point velocities to derive the slip angles.

\section{Tyre Forces and Moments}

The basis for the new tyre modelling is the "Magic Formula" [20-22]. The original development was for car tyres [23], in which context, it has become dominant. The 
extension for motorcycle tyres is relatively recent, with substantial changes being necessary to accommodate the completely different roles of sideslip and camber forces in the two cases. In each case, the "Magic Formula" is a set of equations relating load, slip ratio (longitudinal slip), slip angle and camber angle to longitudinal force, sideforce and aligning moment (and possibly overturning moment), with constraints on the parameters to prevent the behaviour from becoming unreasonable in any operating conditions. Only very limited parameter values can be found in the literature, but a certain amount of relevant experimental data is available. Such data can be used for parameter identification.

A complete set of parameter values for a given tyre will allow the calculation of the steady-state force and moment system for any realistic operating condition. It is required here to determine such a full set of parameters for modern front and rear high performance motorcycle tyres, imposing the condition that the modelled tyres have left/right symmetry. Test data used shows bias and it is necessary to ignore such bias and to omit certain offset terms from the "Magic Formula" relations, in order to model the generic, rather than the particular. Significant published data can be found in [2, 20-25]. Naturally, the older data refers to older tyres, while the newer data relates to contemporary ones. The main sources relied upon here are $[20,23]$. The other sources are used for checking purposes, as appropriate.

\subsection{LONGITUDINAL FORCES IN PURE LONGITUDINAL SLIP}

From Pacejka [23], with the simplifications explained above, the "Magic Formula" expressions for the pure longitudinal slip case are:

$$
\begin{aligned}
\mathrm{d} f_{z} & =\left(F_{z}-F_{z 0}\right) / F_{z 0} \\
F_{x 0} & =D_{x} \sin \left[C_{x} \arctan \left\{B_{x} \kappa-E_{x}\left(B_{x} \kappa-\arctan \left(B_{x} \kappa\right)\right)\right\}\right] \\
D_{x} & =\left(p_{D x 1}+p_{D x 2} \mathrm{~d} f_{z}\right) F_{z} \\
E_{x} & =\left(p_{E x 1}+p_{E x 2} \mathrm{~d} f_{z}+p_{E x 3} \mathrm{~d} f_{z}^{2}\right) \cdot\left(1-p_{E x 4} \operatorname{sgn}(\kappa)\right) \\
K_{x \kappa} & =F_{z}\left(p_{K x 1}+p_{K x 2} \mathrm{~d} f_{z}\right) \cdot \exp \left(p_{K x 3} \mathrm{~d} f_{z}\right) \\
B_{x} & =K_{x \kappa} /\left(C_{x} D_{x}\right)
\end{aligned}
$$

which must satisfy the constraints $D_{x}>0$ and $E_{x}<1$.

Corresponding test results for a 160/70 ZR17 tyre are shown in [23]. The sequential quadratic programming constrained optimisation routine "fmincon" was employed ${ }^{1}$ to iteratively improve the elements of a starting vector of parameters

\footnotetext{
${ }^{1}$ Alternatively, for unconstrained optimization, the Nelder Mead Simplex routine "fminsearch" was employed. Also occasionally, it was necessary to "invent" data, outside the range of experimental results available, to force the identified parameters to give sensible predictions over a wide range of operating circumstances, a problem also referred to in [26]. Often, reasonably accurate starting values for the parameters were needed to ensure convergence to the optimal solution. The methods need to be judged by the results obtained.
} 
Table I. Best-fit parameter values for longitudinal force from 160/70 tyre.

\begin{tabular}{llllllllll}
\hline $\mathrm{C}_{x}$ & $\mathrm{p}_{D x 1}$ & $\mathrm{p}_{D x 2}$ & $\mathrm{p}_{E x 1}$ & $\mathrm{p}_{E x 2}$ & $\mathrm{p}_{E x 3}$ & $\mathrm{p}_{E x 4}$ & $\mathrm{p}_{K x 1}$ & $\mathrm{p}_{K x 2}$ & $\mathrm{p}_{K x 3}$ \\
\hline 1.6064 & 1.2017 & -0.0922 & 0.0263 & 0.27056 & -0.0769 & 1.1268 & 25.94 & -4.233 & 0.3369 \\
\hline
\end{tabular}

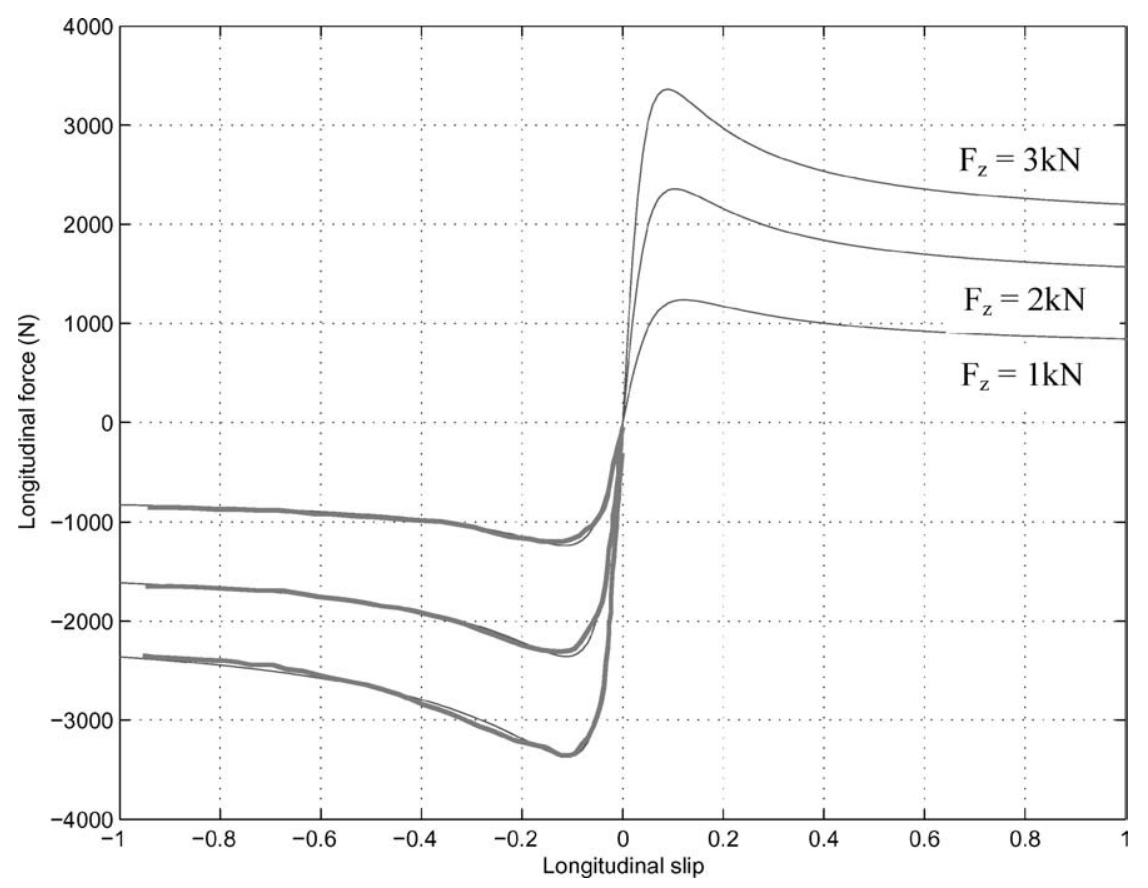

Figure 5. Tyre longitudinal force results for a 160/70 tyre from [23] (thick lines) with best-fit reconstructions (thin lines).

appearing in Equations (1)-(5). The nominal normal load $F_{z 0}$ was chosen to be $1600 \mathrm{~N}$ based on typical usage of such a tyre. That choice is far from critical, in fact, a change leading to compensatory changes in other parameters. Optimal parameters are given in Table I and the fits are illustrated in Figure 5. The two constraints are satisfied for loads less than $20890 \mathrm{~N}$, which includes all practical circumstances.

Longitudinal force results are not available for any other tyres, so lateral forces are considered next.

\subsection{LATERAL FORCES IN PURE SIDESLIP AND CAMBER}

In exactly the same way, the relevant equations for the lateral force are:

$$
\begin{aligned}
F_{y 0}= & D_{y} \sin \left[C_{y} \arctan \left\{B_{y} \beta-E_{y}\left(B_{y} \beta-\arctan \left(B_{y} \beta\right)\right)\right\}\right. \\
& \left.+C_{\gamma} \arctan \left\{B_{\gamma} \gamma-E_{\gamma}\left(B_{\gamma} \gamma-\arctan \left(B_{\gamma} \gamma\right)\right)\right\}\right]
\end{aligned}
$$



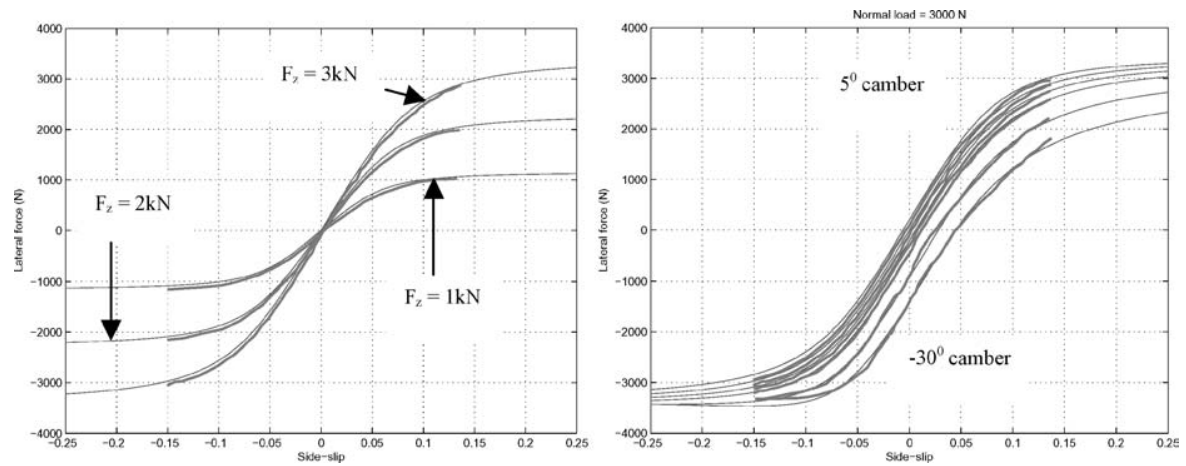

Figure 6. Tyre lateral force results for a 160/70 tyre from [23] (thick lines) with best-fit reconstructions (thin lines). Camber angles $5,0,-5,-10,-20,-30^{\circ}$.

$$
\begin{aligned}
D_{y}= & F_{z} p_{D y 1} \exp \left(p_{D y 2} \mathrm{~d} f_{z}\right) /\left(1+p_{D y 3} \gamma^{2}\right) \\
E_{y}= & p_{E y 1}+p_{E y 2} \gamma^{2}+p_{E y 4} \gamma \operatorname{sgn}(\beta) \\
K_{y \alpha}= & p_{K y 1} F_{z 0} \sin \left[p_{K y 2} \arctan \left\{F_{z} /\left(\left(p_{K y 3}+p_{K y 4} \gamma^{2}\right) F_{z 0}\right)\right\}\right] \\
& /\left(1+p_{K y 5} \gamma^{2}\right) \\
B_{y}= & K_{y \alpha} /\left(C_{y} D_{y}\right) \\
K_{y \gamma}= & \left(p_{K y 6}+p_{K y 7} \mathrm{~d} f_{z}\right) F_{z} \\
B_{\gamma}= & K_{y \gamma} /\left(C_{\gamma} D_{y}\right)
\end{aligned}
$$

with the constraints $C_{y}+C_{\gamma}<2, C_{y}>0, D_{y}>0, E_{y}<1, C_{g}>0, E_{g}<1$. For the same tyre as before, the parameter optimisation process, with the effective friction coefficient limited to values no greater than 1.3, gives the results illustrated in Figure 6 with parameter values given below in Table II. For this particular tyre, $p_{K y}$ in Equation (12) was set to zero, because experimental results are only available

Table II. Best-fit parameter values for lateral force from 160/70 (top), 120/70 (middle) and $180 / 55$ (bottom) tyres

\begin{tabular}{llllllll}
\hline $\mathrm{C}_{y}$ & $\mathrm{p}_{D y 1}$ & $\mathrm{p}_{D y 2}$ & $\mathrm{p}_{D y 3}$ & $\mathrm{p}_{E y 1}$ & $\mathrm{p}_{E y 2}$ & $\mathrm{p}_{E y 4}$ & $\mathrm{p}_{K y 1}$ \\
\hline 0.93921 & 1.1524 & -0.01794 & -0.06531 & -0.94635 & -0.09845 & -1.6416 & 26.601 \\
0.8327 & 1.3 & 0 & 0 & -1.2556 & -3.2068 & -3.998 & 22.841 \\
0.9 & 1.3 & 0 & 0 & -2.2227 & -1.669 & -4.288 & 15.791 \\
$\mathrm{p}_{K y 2}$ & $\mathrm{p}_{K y 3}$ & $\mathrm{p}_{K y 4}$ & $\mathrm{p}_{K y 5}$ & $\mathrm{C}_{\gamma}$ & $\mathrm{p}_{K y 6}$ & $\mathrm{p}_{K y 7}$ & $\mathrm{E}_{\gamma}$ \\
1.0167 & 1.4989 & 0.52567 & -0.24064 & 0.50732 & 0.7667 & 0 & -4.7481 \\
2.1578 & 2.5058 & -0.08088 & -0.22882 & 0.86765 & 0.69677 & -0.03077 & -15.815 \\
1.6935 & 1.4604 & 0.669 & 0.18708 & 0.61397 & 0.45512 & 0.013293 & -19.99 \\
\hline
\end{tabular}



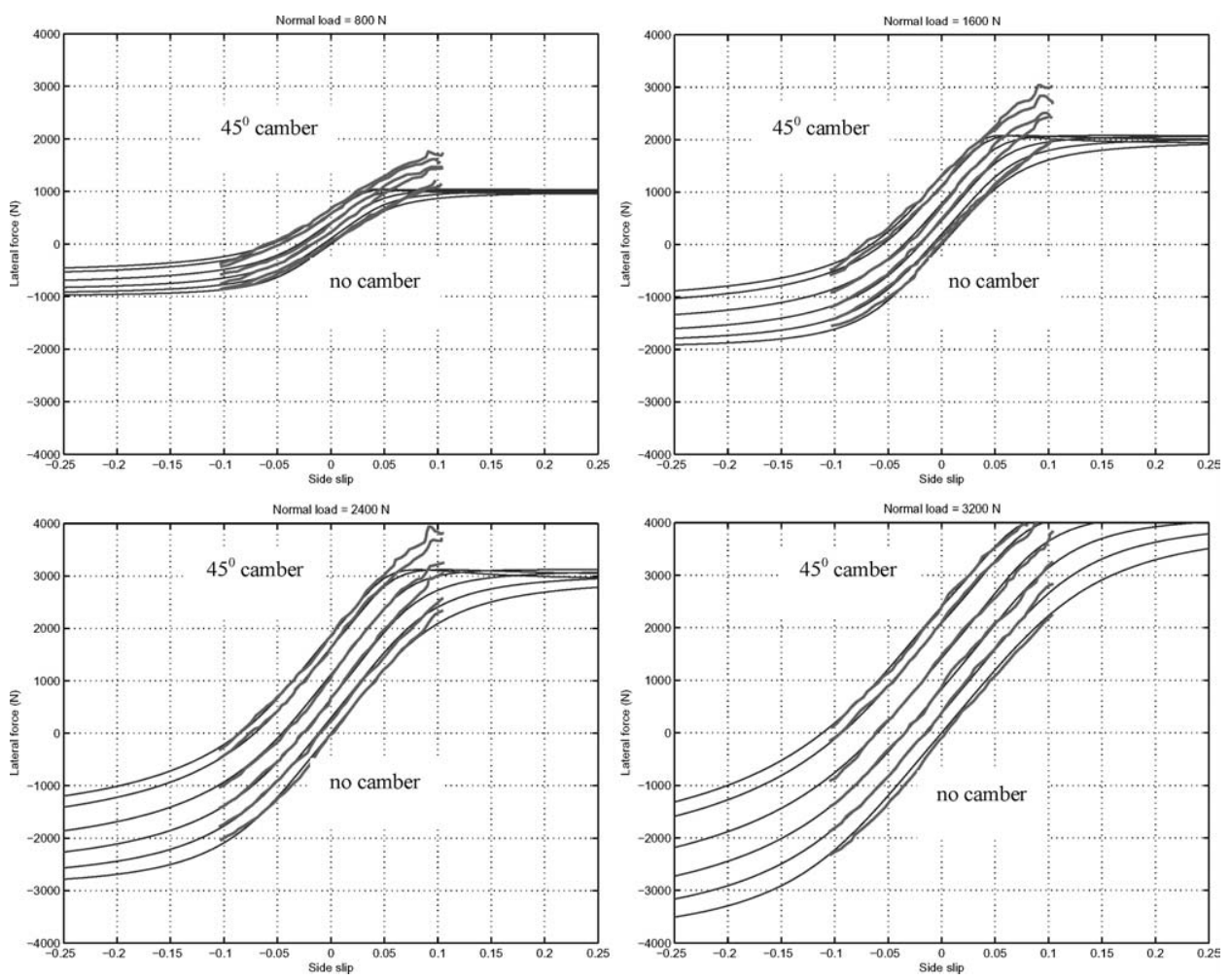

Figure 7. Tyre lateral force results for 120/70 tyre from [20] (thick lines) with best-fit reconstructions (thin lines). Camber angles $0,10,20,30,40,45^{\circ}$.

at non-zero camber angle for one load. This is consistent with results obtained for $120 / 70$ and $180 / 55$ tyres (see below), for which $p_{K y 7}$ is relatively small, being positive in one case and negative in the other. All the constraints are satisfied for camber angles less than $70^{\circ}$ in magnitude.

Next, the lateral force fitting is repeated for the experimental results included in [20] for a 120/70 front tyre and a 180/55 rear tyre, first recognising that the former results suffer from an unreasonable positive force offset, especially for the smaller loads, which would imply a friction coefficient greater than 2 , if they were true. To avoid responding too strongly to these apparently spurious features, $D_{y}$ is allowed to be no greater than 1.3 times $F_{z}$. Also, the measurements for slip angles greater than $+5^{\circ}$ are ignored. The previous rear tyre value of $F_{z 0}$ as $1600 \mathrm{~N}$ is retained while the non-critical value for the front tyre was chosen as $1100 \mathrm{~N}$. Bestfit parameters are shown in Table II, with Figures 7 and 8 showing the quality of the fits for the front and rear tyres respectively. All the constraints are satisfied by these parameters. 

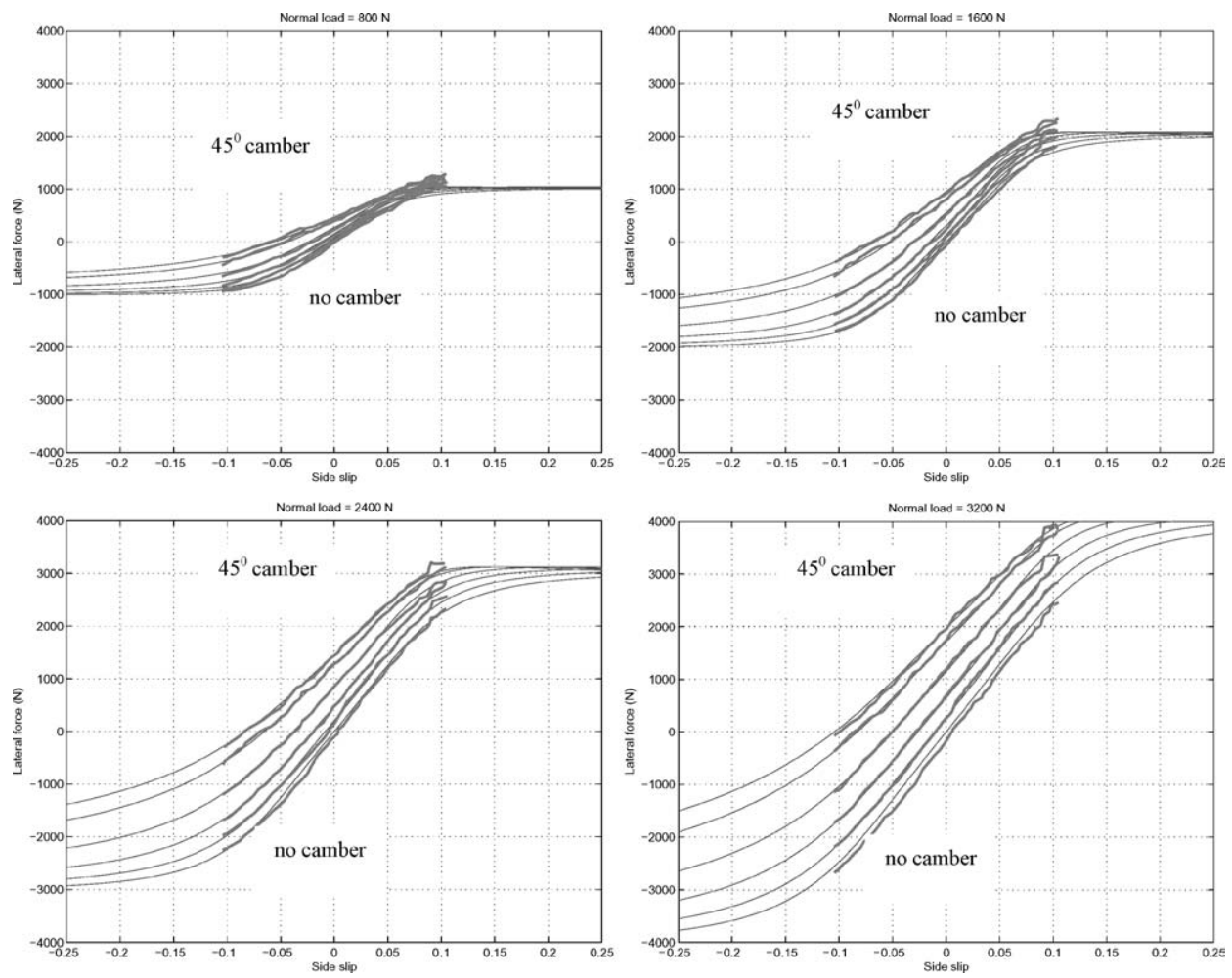

Figure 8. Tyre lateral force results for 180/55 tyre from [20] (thick lines) with best-fit reconstructions (thin lines). Camber angles 0, 10, 20, 30, 40, 45 .

\subsection{ALIGNING MOMENTS IN LATERAL SLIP AND CAMBER}

Aligning moment results are included in [23] for the 160/70 tyre and in [20] for $120 / 70$ and $180 / 55$ tyres. Three loads are covered in the former but only two in the latter, which makes the model very heavy in parameters for the amount of experimental data available. In setting the parameters for the 160/70 tyre of [23] assuming the full quadratic dependency of $B_{t}$ on load, the fitting is good within the load range used for the measurements but the extrapolation is poor, with constraint violations at low and high loads. With linear dependency, the fitting is almost as good and the extrapolation problem can be eliminated. Consequently, $B_{t}$ is considered linear with load. Even so, there are many parameter combinations which give almost equally good fits to the limited data. It is advantageous to use some physical reasoning to guide the choice between the alternatives. The product of $B_{t}, C_{t}$ and $D_{t}$ is the aligning moment stiffness of the tyre. According to the "Brush Model" [23], the aligning moment stiffness is proportional to load to the power 1.5, so that feature is used to aid the choice of the secondary parameters $q_{B z 1}$ and $q_{B z 2}$, see (18). It turns out to be quite feasible to match that characteristic closely. Also, as before, 
Table III. Best-fit parameter values for aligning moment from 160/70 (top), 120/70 (middle) and 180/55 tyre (bottom)

\begin{tabular}{ccccccc}
\hline $\mathrm{C}_{t}$ & $\mathrm{q}_{B z 1}$ & $\mathrm{q}_{B z 2}$ & $\mathrm{q}_{B z 5}$ & $\mathrm{q}_{B z 6}$ & $\mathrm{q}_{B z 9}$ & $\mathrm{q}_{B z 10}$ \\
\hline 1.3115 & 10.354 & 4.3004 & -0.34033 & -0.13202 & 10.118 & -1.0508 \\
1.0917 & 10.486 & -0.001154 & -0.68973 & 1.0411 & 27.445 & -1.0792 \\
1.3153 & 10.041 & $-1.61 \mathrm{e}-8$ & -0.76784 & 0.73422 & 16.39 & -0.35549 \\
$\mathrm{q}_{D z 1}$ & $\mathrm{q}_{D z 2}$ & $\mathrm{q}_{D z 3}$ & $\mathrm{q}_{D z 4}$ & $\mathrm{q}_{D z 8}$ & $\mathrm{q}_{D z 9}$ & $\mathrm{q}_{D z 10}$ \\
0.20059 & 0.05282 & -0.21116 & -0.15941 & 0.30941 & 0 & 0.10037 \\
0.19796 & 0.06563 & 0.2199 & 0.21866 & 0.3682 & 0.1218 & 0.25439 \\
0.26331 & 0.030987 & -0.62013 & 0.98524 & 0.50453 & 0.36312 & -0.19168 \\
$\mathrm{q}_{D z 11}$ & $\mathrm{q}_{E z 1}$ & $\mathrm{q}_{E z 2}$ & $\mathrm{q}_{E z 5}$ & $\mathrm{q}_{H z 3}$ & $\mathrm{q}_{H z}$ & \\
0 & -3.9247 & 10.809 & 0.9836 & -0.04908 & 0 & \\
-0.17873 & -0.91586 & 0.11625 & 1.4387 & -0.003789 & -0.01557 & \\
-0.40709 & -0.19924 & -0.017638 & 3.6511 & -0.028448 & -0.009862 & \\
\hline
\end{tabular}

right/left symmetry and zero offsets are assumed, making $q_{E z 4}, q_{H z 1}$ and $q_{H z 2}$ zero.

The relevant "Magic Formula" Equations [23] are:

$$
\begin{aligned}
M_{z 0}= & M_{z t 0}+M_{z r 0} \\
M_{z t 0}= & -D_{t} \cos \left[C_{t} \arctan \left\{B_{t} \beta-E_{t}\left(B_{t} \beta-\arctan \left(B_{t} \beta\right)\right)\right\}\right] \\
& / \sqrt{1+\beta^{2}} \cdot F_{y 0, \gamma=0} \\
M_{z r 0}= & D_{r} \cos \left[\arctan \left(B_{r}\left(\beta+S_{H r}\right)\right]\right. \\
S_{H r}= & \left(q_{H z 3}+q_{H z 4} \mathrm{~d} f_{z}\right) \gamma \\
B_{t}= & \left(q_{B z 1}+q_{B z 2} \mathrm{~d} f_{z}\right)\left(1+q_{B z 5}|\gamma|+q_{B z 6} \gamma^{2}\right) \\
D_{t}= & F_{z}\left(R_{0} / F_{z 0}\right)\left(q_{D z 1}+q_{D z 2} \mathrm{~d} f_{z}\right)\left(1+q_{D z 3}|\gamma|+q_{D z 4} \gamma^{2}\right) \\
E_{t}= & \left(q_{E z 1}+q_{E z 2} \mathrm{~d} f_{z}\right)\left\{1+q_{E z 5} \gamma(2 / \pi) \arctan \left(B_{t} C_{t} \beta\right)\right\} \\
B_{r}= & q_{B z 9}+q_{B z 10} B_{y} C_{y} \\
D_{r}= & F_{z} R_{0}\left\{\left(q_{D z 8}+q_{D z 9} \mathrm{~d} f_{z}\right) \gamma+\left(q_{D z 10}+q_{D z 11} \mathrm{~d} f_{z}\right) \gamma|\gamma|\right\} \\
& \quad / \sqrt{1+\beta^{2}}
\end{aligned}
$$

with the constraints: $B_{t}>0, C_{t}>0$ and $E_{t}<1$. For the 160/70 tyre, $q_{H z 4}$ in Equation (17) and $q_{D z 9}$ and $q_{D z 11}$ in Equation (22) are set to zero, because experimental results are only provided at non-zero camber angle for one load.

The tyre crown radius, $R_{0}$, for each tyre derives from the cross-sectional geometry as $0.08 \mathrm{~m}$ for $160 / 70,0.06 \mathrm{~m}$ for $120 / 70$ and $0.09 \mathrm{~m}$ for $180 / 55$ [7]. Identification of the remaining parameters using "fmincon" as before gives the values in Table III. Constraint violations occur only for loads greater than $11 \mathrm{kN}$, sideslip angle greater than $45^{\circ}$ or camber angle greater than $60^{\circ}$. These violations are outside the practical running range. The fit qualities are shown in Figures 9-11. 

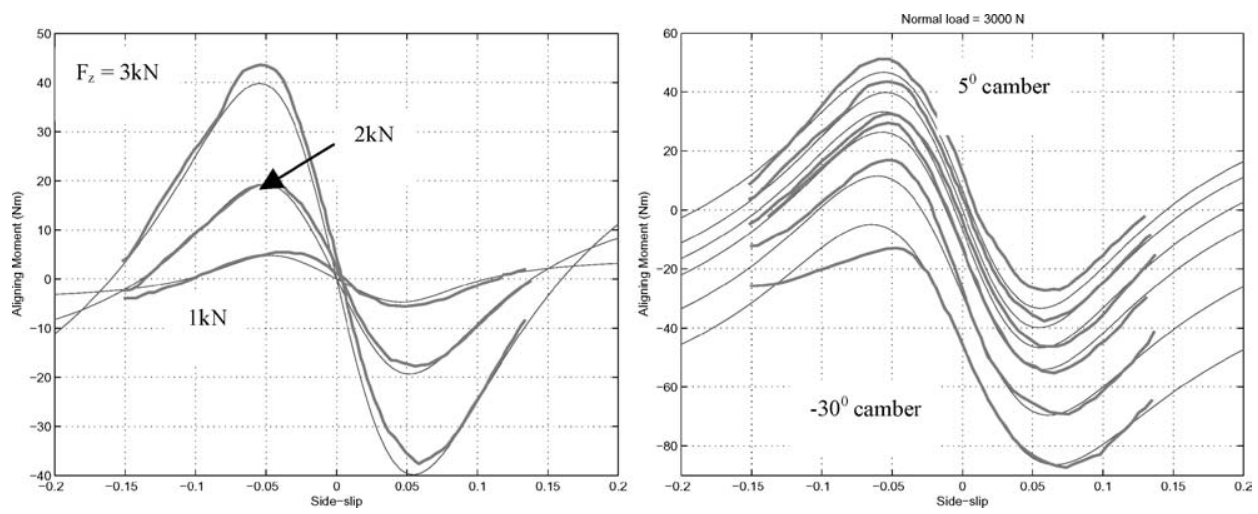

Figure 9. Tyre aligning moment results for 160/70 tyre from [23] (thick lines) with best-fit reconstructions (thin lines). Camber angles $5,0,-5,-10,-20,-30^{\circ}$.
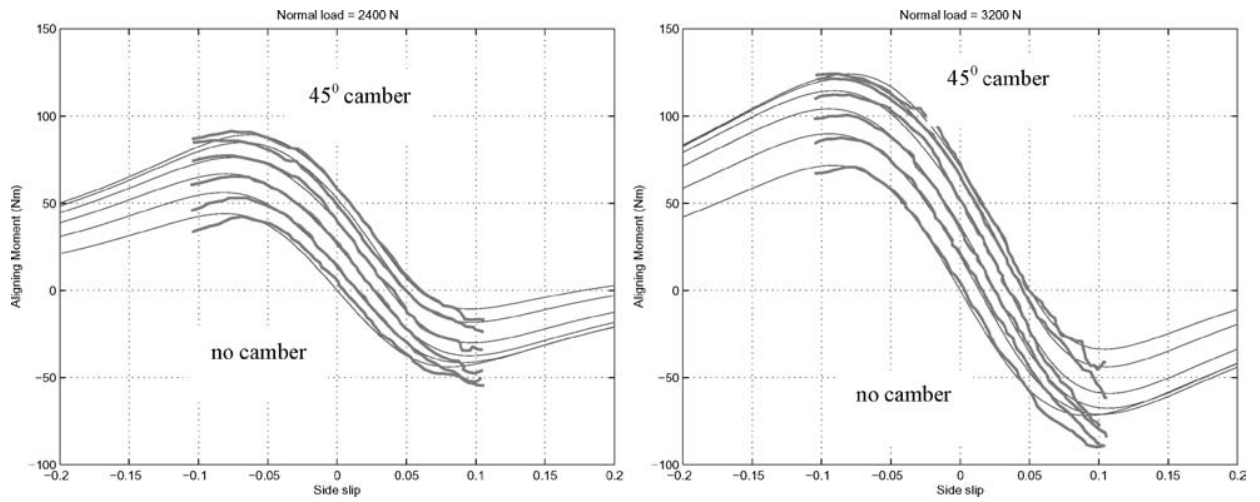

Figure 10. Tyre aligning moment results for 120/70 tyre from [20] (thick lines) with best-fit reconstructions (thin lines). Camber angles $0,10,20,30,40,45^{\circ}$.
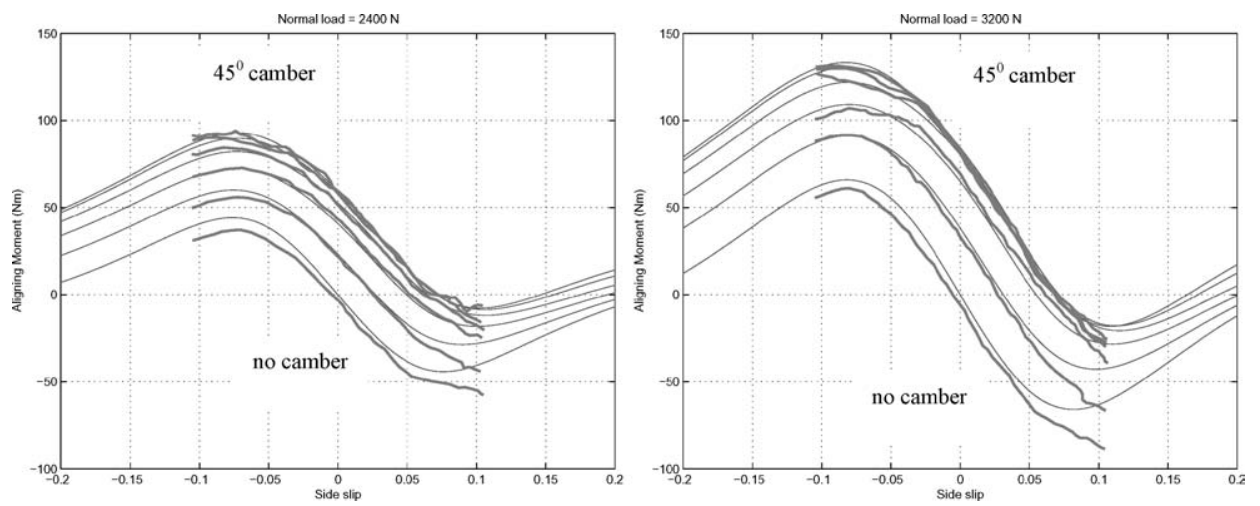

Figure 11. Tyre aligning moment results for 180/55 tyre from [20] (thick lines) with best-fit reconstructions (thin lines). Camber angles $0,10,20,30,40$ and $45^{\circ}$. 


\subsection{COMBINED SLIP RESULTS}

\subsubsection{Longitudinal Forces}

In the "Magic Formula" scheme, the loss of longitudinal force due to sideslipping is described by a "loss function" to be applied to the pure slip force described above. Presuming as before that the generic tyres of interest will be symmetric $\left(S_{H x \alpha}=0\right)$ and, in the absence of any indication to the contrary, assuming that wheel camber will not affect the loss of longitudinal force due to sideslipping $\left(r_{B \times 3}=0\right)$, the equations describing the loss are:

$$
\begin{aligned}
F_{x} & =\cos \left[C_{x \alpha} \arctan \left(B_{x \alpha} \beta\right)\right] F_{x 0} \\
B_{x \alpha} & =r_{B x 1} \cos \left[\arctan \left(r_{B x 2} \kappa\right)\right]
\end{aligned}
$$

with the constraints that $F_{x}>0$ and $B_{x \alpha}>0$.

The only relevant combined slip data available is from [23] for the 160/70 tyre for $3 \mathrm{kN}$ load and zero camber angle. The same parameter identification process as before yielded the best values as $r_{B x 1}=13.476 ; r_{B x 2}=11.354 ; C_{x \alpha}=1.1231$, with the fit quality shown in Figure 12 . The constraint on $B_{x \alpha}$ is always satisfied while that on $F_{x}$ is satisfied for slip angles less than $23^{\circ}$, which is considered to provide an adequate operating range.

\subsubsection{Lateral Forces}

In the same way (with $S_{V y \kappa}=S_{H y \kappa}=r_{B y 4}=0$ ), the equations describing the loss of lateral force due to longitudinal slip are:

$$
\begin{aligned}
F_{y} & =\cos \left[C_{y \kappa} \arctan \left(B_{y \kappa} \kappa\right)\right] F_{y 0} \\
B_{y \kappa} & =r_{B y 1} \cos \left[\arctan \left\{r_{B y 2}\left(\beta-r_{B y 3}\right)\right\}\right]
\end{aligned}
$$

with constraints $F_{y}>0$ and $B_{y k}>0$.

Data again comes from Pacejka [23] and is for the 160/70 tyre at $3 \mathrm{kN}$ and zero camber. It yields the best-fit parameters as $r_{B y 1}=7.7856, r_{B y 2}=8.1697, r_{B y 3}=$ -0.05914 and $C_{y \kappa}=1.0533$. The fit quality is shown in Figures 13 and 14 .

\subsubsection{Aligning Moments}

The relevant equations (with $s=S_{V y \kappa}=S_{H y \kappa}=0$ ) are:

$$
\begin{aligned}
M_{z}= & -D_{t} \cos \left[C_{t} \arctan \left\{B_{t} \lambda_{t}-E_{t}\left(B_{t} \lambda_{t}-\arctan \left(B_{t} \lambda_{t}\right)\right)\right\}\right] \\
& / \sqrt{1+\beta^{2}} \cdot F_{y, \gamma=0}+M_{z r} \\
F_{y, \gamma=0}= & \cos \left[C_{y \kappa} \arctan \left(B_{y \kappa} \kappa\right)\right] \cdot F_{y 0, \gamma=0} \\
M_{z r}= & D_{r} \cos \left[\arctan \left(B_{r} \lambda_{r}\right)\right] \\
\lambda_{t}= & \sqrt{\beta^{2}+\left(K_{x \kappa} \kappa / K_{y \alpha, \gamma=0}\right)^{2}} \operatorname{sgn}(\beta) \\
\lambda_{r}= & \sqrt{\left(\beta+S_{H r}\right)^{2}+\left(K_{x \kappa} \kappa / K_{y \alpha, \gamma=0}\right)^{2}} \operatorname{sgn}\left(\beta+S_{H r}\right)
\end{aligned}
$$




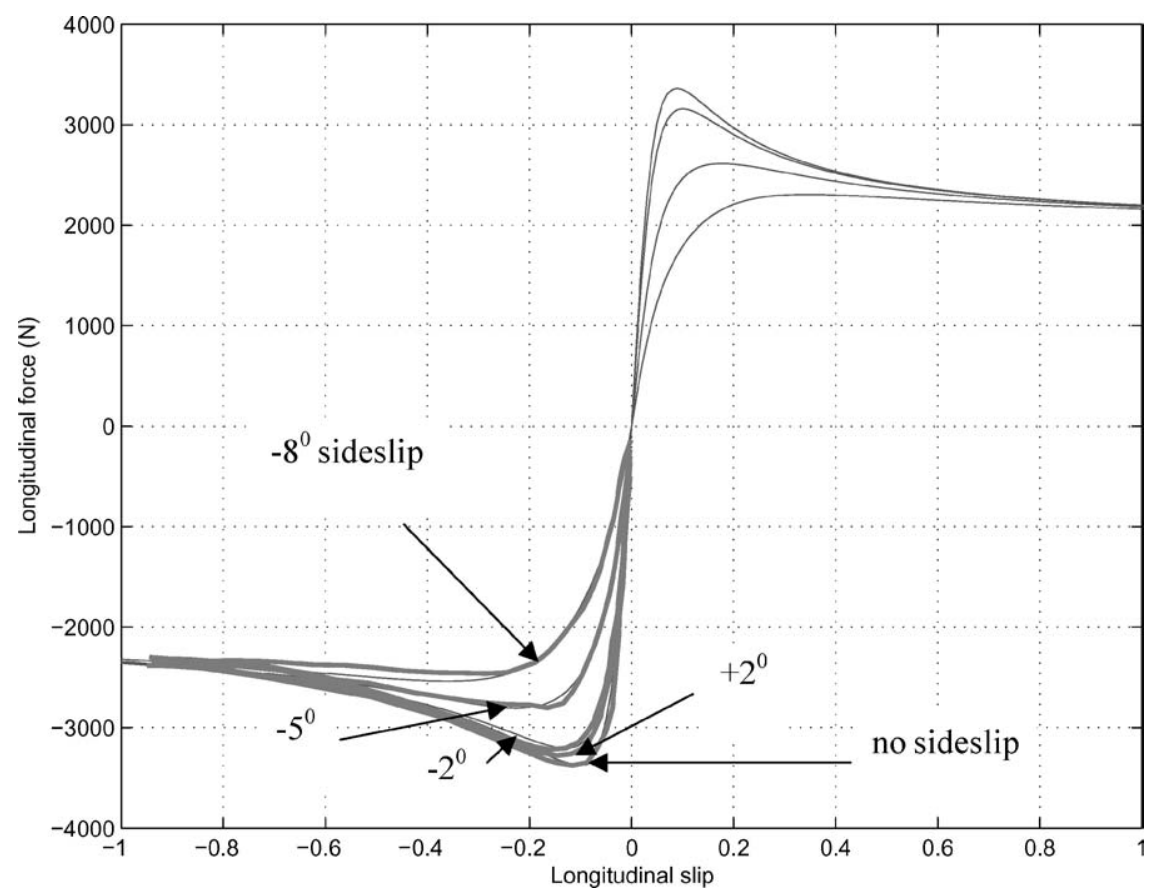

Figure 12. Influence of sideslip on longitudinal force for $160 / 70$ tyre at $3 \mathrm{kN}$ load and zero camber from [23] (thick lines) with best-fit reconstructions (thin lines). Theoretical curves for +2 and $-2^{\circ}$ camber are indistinguishable.

The term $s \cdot F_{x}$ in the original [23] is omitted, since $s$ here is zero, by virtue of the reference point for moments being the actual contact point.

Expressions for $K_{x \kappa}, F_{y 0}, K_{y \alpha}, B_{t}, E_{t}, D_{r}, C_{y k}$ and $B_{y \kappa}$ are given by (5), (7), (10), (18), (20), (21), (22), (25) and (26) respectively, and $C_{t}$ is given in Table III. Thus further parameter identification is unnecessary and the combined slip moments can be predicted from what is known already. The aligning moment for the 160/70 tyre at $3 \mathrm{kN}$ load, as a function of longitudinal slip, for several slip angles, is shown in Figure 15.

\subsection{LONGITUDINAL FORCE MODELS FOR 120/70 AND 180/55 TYRES}

Longitudinal forces for 120/70 and 180/55 tyres were not measured in [20]. In order to complete a general description of those tyres, it is necessary to make up, using the best evidence available, appropriate parameter values to describe their properties. The strategy for doing this is to use the 160/70 tyre as a model and to scale its data to obtain those for the other tyres. Longitudinal pure slip parameters for the 160/70 tyre are given above in Table I, while those for pure lateral slip appear in Table II. In particular, the ratio of peak forces $D_{x} / D_{y}$ is evaluated 


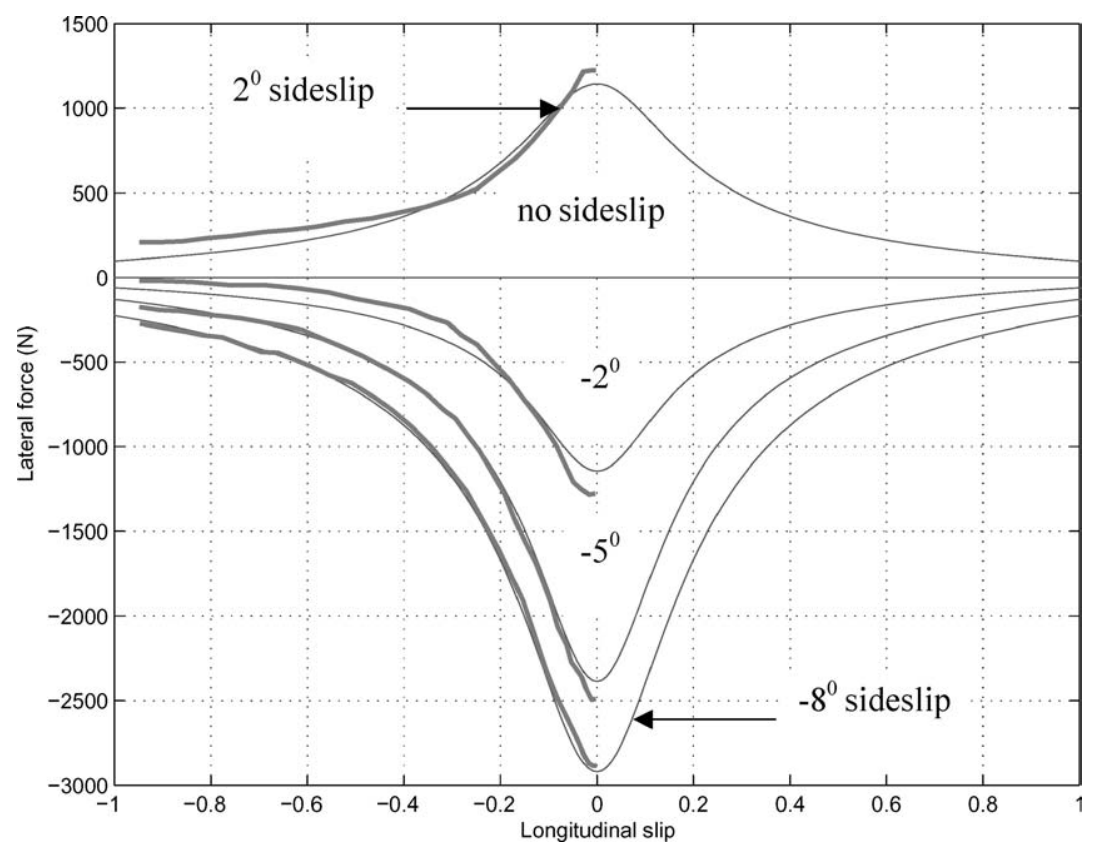

Figure 13. Influence of sideslip on lateral force for 160/70 tyre at $3 \mathrm{kN}$ load and zero camber from [23] (thick lines) with best-fit reconstructions (thin lines).

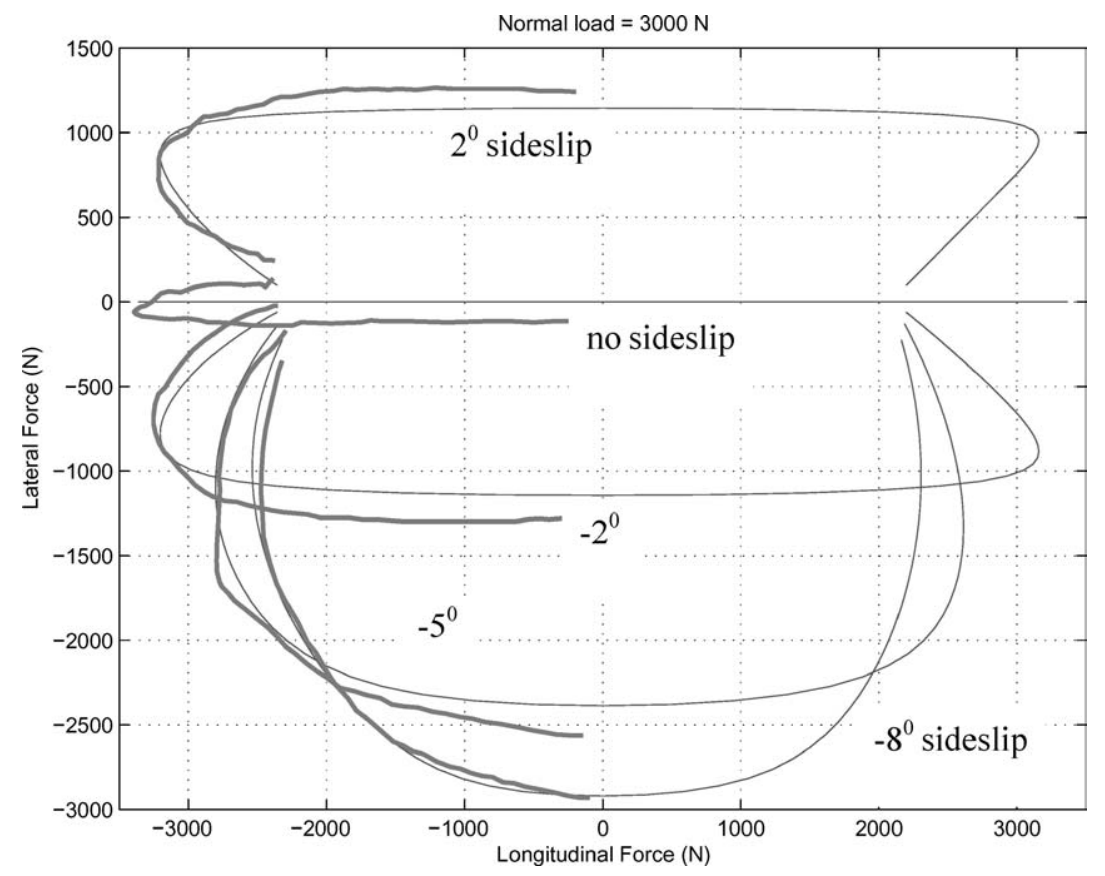

Figure 14. Influence of sideslip on longitudinal and lateral forces for $160 / 70$ tyre at $3 \mathrm{kN}$ load and zero camber from [23] (thick lines) with best-fit reconstructions (thin lines). 


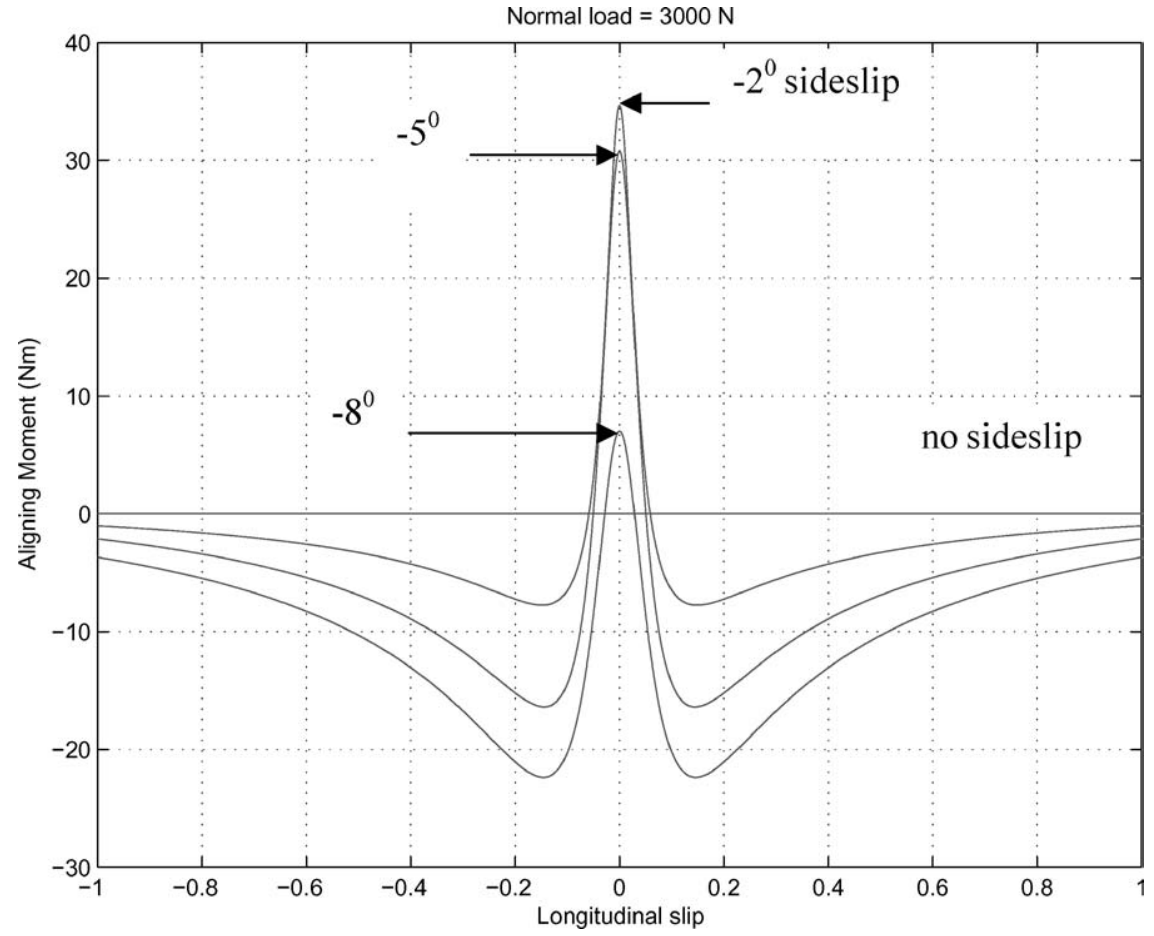

Figure 15. Aligning moment for $160 / 70$ tyre at $3 \mathrm{kN}$ load and zero camber as a function of longitudinal slip for each of four sideslip cases.

for 1,2 and $3 \mathrm{kN}$ load as 1.066, 1.028 and 0.989 respectively. The same ratios are assumed to apply to the $120 / 70$ and $180 / 55$ tyres, with their shapes assumed to be the same as those for the 160/70 tyre. The only new parameters needed are $p_{D x 1}$ and $p_{D x 2}$, with values 1.381 for $120 / 70$ and 1.355 for $180 / 55$ and -0.04143 for $120 / 70$ and -0.0603 for $180 / 55$ respectively. Longitudinal force peaks are about 1.33 times the tyre load in the usual operating range of loads, which is compatible with acceleration and braking performances obtainable from a contemporary motorcycle. Parameters apart from $p_{D x 1}$ and $p_{D x 2}$ in Table I apply to this case.

\subsection{COMBINED SLIP FORCE MODELS FOR 120/70 AND 180/55 TYRES}

In the same way, the combined slip parameters given for the 160/70 tyre in Sections 4.4.1 and 4.4.2 are regarded as describing the behaviour of the 120/70 and 180/55 tyres also. Combination of longitudinal force under pure longitudinal slip with the loss function data from Section 4.4.1 and of lateral force under pure lateral slip with the loss function data of Section 4.4.2 allows the prediction of combined slip forces generally. 


\subsection{CHECKING AGAINST OTHER DATA}

The complete tyre model has been used to calculate the force and moment system corresponding to running conditions for which data has been published $[2,7,24,25$, $27,28]$. In each case, the results compare reasonably with the originals, providing reassurance that the generic model with its parameter values can be employed with confidence.

\subsection{RELAXATION LENGTH DESCRIPTION AND DATA}

To replicate the properties of the higher frequency modes in particular, it is essential to model motorcycle tyres with relaxation lags included [1]. Conventionally, a constant relaxation length for each tyre is employed but it was found in [20] that the tyre relaxation length typically varies with load roughly as the cornering stiffness does and that it grows with speed. Using the data from [20] for 120/70 front and $180 / 55$ rear tyres and fitting a quadratic function of speed to the results in each case, we obtain the descriptions:

$$
\sigma_{f}=K_{y \alpha f}\left(8.633 \mathrm{e}-6+3.725 \mathrm{e}-8 . V+8.389 \mathrm{e}-10 . V^{2}\right)
$$

and

$$
\sigma_{r}=K_{y \alpha r}\left(9.694 \mathrm{e}-6-1.333 \mathrm{e}-8 V+1.898 \mathrm{e}-9 V^{2}\right)
$$

The cornering stiffnesses come out of the "Magic Formula" computations, Equation (11). Relaxation is applied to the sideslip rather than the sideforces, through equations of the form: $\sigma \dot{\beta}_{1} / V+\beta_{1}=\beta$. This implies that forces and moments arising from wheel camber are treated as occurring without delay, while those arising from sideslip are lagged. This is considered to be the most physically accurate representation, since camber leads to forces geometrically while sideslip leads to forces via distortion of the tyre carcass, which distortion requires time (or distance rolled) to establish.

\section{5. "Monoshock" Rear Suspension}

The motorcycle rear suspension arrangement is shown diagrammatically in Figure 16. It uses a single spring/damper unit with a mechanical linkage connection to the swinging arm. Many modern rear suspensions are of this type, although several variants of it exist. It involves a closed kinematic loop. Such a suspension can be modelled on-line literally, link by link and joint by joint, or off-line, via a separate geometric pre-analysis. Such a pre-analysis yields an analytic relationship between the swing arm angle change and the moment of the spring force about the swing arm pivot, which is used directly in the multibody model building. Alternatively, if the pre-analysis were too complex to give an analytic result, a numerical relationship 


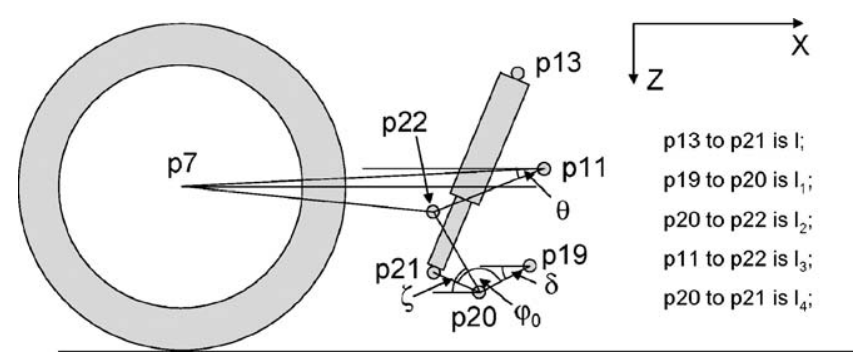

Figure 16. Geometry of monoshock suspension arrangement on GSX-R1000 motorcycle. Distances between various points are also defined in the diagram.

between the angle and the moment could be found. This could be replaced by an approximate functional relationship covering the practical range of the swing arm movement. A low order polynomial will usually suffice [29]. The literal modelling is the simpler, but it will provide equations of motion which integrate relatively slowly, since the simulation has to solve the kinematic loop equations at each integration step. The analysis follows.

Points $\mathrm{p} 11, \mathrm{p} 13$ and $\mathrm{p} 19$ are fixed to the main frame. $l_{1}, l_{4}$ and $\varphi_{0}$ are dimensions of the swinging link and $l_{2}$ the length of the pull rod. The length $l_{3}$ in the swing arm is fixed. The spring/damper unit is of variable length $l . \theta$ is the angle of the swing arm to the horizontal $x$-axis, while $\delta$ is the corresponding angle for the swinging link. Traversing the loop p11-p22-p20-p19-p11, both $x$ and $z$ displacements are nil, since we end where we begin. Therefore:

$$
\begin{array}{ll} 
& x_{11}-x_{19}-l_{3} \cos \theta+l_{2} \cos \zeta+l_{1} \cos \delta=0 \\
\text { and } & z_{11}-z_{19}+l_{3} \sin \theta+l_{2} \sin \zeta-l_{1} \sin \delta=0
\end{array}
$$

Forming $l_{2}^{2}$ as $\left(l_{2}^{2} \sin ^{2} \zeta+l_{2}^{2} \cos ^{2} \zeta\right)$ and substituting:

$$
c_{1}=-x_{11}+x_{19}+l_{3} \cos \theta \text { and } c_{2}=-z_{11}+z_{19}-l_{3} \sin \theta
$$

we obtain: $l_{2}^{2}=\left(c_{1}-l_{1} \cos \delta\right)^{2}+\left(c_{2}-l_{1} \sin \delta\right)^{2}$ from which it can be shown that:

$$
\delta=\arcsin \left(\frac{l_{2}^{2}-l_{1}^{2}-c_{1}^{2}-c_{2}^{2}}{2 l_{1} \sqrt{c_{1}^{2}+c_{2}^{2}}}\right)+\arctan \left(\frac{c_{1}}{c_{2}}\right),
$$

which is a function of $\theta$ only. Also:

$$
\text { and } \quad \begin{aligned}
x_{21} & =x_{19}-l_{1} \cos \delta+l_{4} \cos \left(\phi_{0}+\delta\right) \\
z_{21} & =z_{19}+l_{1} \sin \delta-l_{4} \sin \left(\phi_{0}+\delta\right)
\end{aligned}
$$




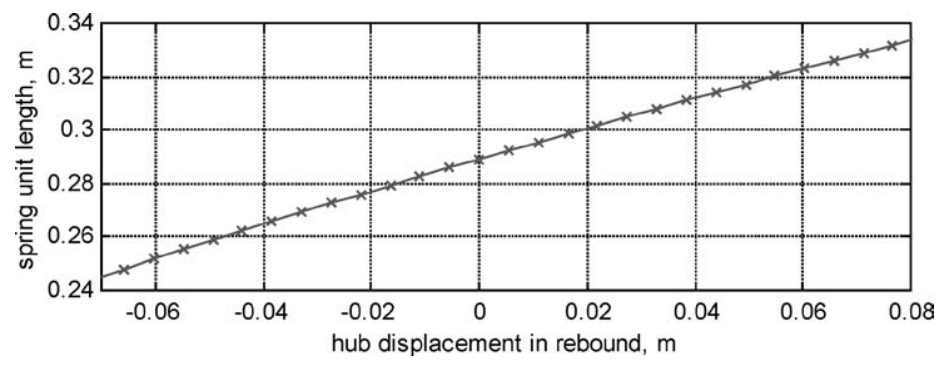

Figure 17. Spring / damper unit length to wheel displacement relationship for GSX-R1000 motorcycle.

with

$$
l=\sqrt{\left(x_{13}-x_{21}\right)^{2}+\left(z_{13}-z_{21}\right)^{2}}
$$

so that $l$ can be found as a function of $\theta, l=f_{1}(\theta)$ say, by substitution for $x_{21}$ and $z_{21}$ in this expression. Figure 17 illustrates the outcome. If a small change $\delta \theta$ in $\theta$ occurs, in which the corresponding change in $l$ is $\delta l$, the moment $M$ corresponding to a spring/damper force $f_{2}(l, \dot{l})$ is $f_{2}(l, \dot{l}) \cdot \mathrm{d} l / \mathrm{d} \theta$ by virtual work. The properties of the spring/damper unit can thus be expressed in terms of an equivalent moment $M(\theta, \dot{\theta})$ about the swing-arm pivot, as:

$$
M=f_{2}\left\{f_{1}(\theta), \frac{\mathrm{d} f_{1}(\theta)}{\mathrm{d} \theta} \dot{\theta}\right\} \frac{\mathrm{d} f_{1}(\theta)}{\mathrm{d} \theta},
$$

which can be fully automated.

\section{Speed and Steer Controllers}

To maintain a desired forward speed profile, driving torque is applied to the rear wheel and reacted on the main frame. The torque is produced by a proportional/integral control on the speed error with fixed gains. Although the reference machine has a chain drive to the rear wheel, this representation is of a shaft drive system and it needs updating to deal with issues like the prediction of suspension movements and body attitudes under heavy acceleration. For milder longitudinal manoeuvring, there will be little difference between shaft drive and chain drive. The target speed is provided as data in a table function, with time as the independent variable.

A steering feedback controller is also necessary to stabilise the machine in manoeuvres in which it is not self-stable. In particular, stabilising control allows the solution of the steady turning equilibrium state problem by simply running a simulation to steady state. The controller devised is a proportional/integral/derivative 
(PID) feedback of motorcycle lean angle error to steering torque, with the lean angle target being set by an initial value and a constant rate of change. The target lean angle must therefore be a ramp function of time. This would be easy to alter if it were considered restrictive.

The steering control gains need to be speed adaptive and they need choosing with considerable care to achieve effective stabilisation. Especially difficult are cases involving very low or very high speed and high lean angles. Each of the three PID gains is linearly related to speed, as indicated by the relations:

$$
G_{p}=\operatorname{spg} 0+\operatorname{spg} 1 \cdot u ; G_{i}=\operatorname{sig} 0+\operatorname{sig} 1 \cdot u ; G_{d}=\operatorname{sdg} 0+\operatorname{sdg} 1 \cdot u ;
$$

corresponding to the control law:

$$
\tau=G_{p}\left(\phi-\phi_{\mathrm{ref}}\right)+G_{i} \int_{0}^{t}\left(\phi-\phi_{\mathrm{ref}}\right) \mathrm{d} t+G_{d} \dot{\phi} ;
$$

where $u$ is the forward speed, $\tau$ is the steering control torque, $\phi$ is the lean angle and $\phi_{\text {ref }}$ is the target lean angle.

\section{Equilibrium State Checking and Power Balancing}

With suitable stabilisation, the motorcycle can be run to equilibrium at any feasible speed and lean angle. To describe such an equilibrium state, force and moment balance equations can be set up, as was done in [5, 30]. As described in [30], the checking process includes a power balance, whereby the engine power is shown to account precisely for the aerodynamic and tyre losses. In steady turning, the force balance check is to ensure that the sum of the external forces is equal to the sum of the inertial and gravitational forces. The force error calculated is:

$$
\underline{F}_{\text {error }}=\sum_{i} \underline{F}_{i}+\sum_{j} m_{j}\left(\underline{g}-\underline{\omega}_{j} \times \underline{v}_{j}\right)
$$

the first sum containing all the external forces, while the second deals with gravitational and centripetal effects. The external forces include: (i) aerodynamic lift and drag forces, (ii) the front and rear wheel normal loads, (iii) the tyre side forces and (iv) the tyre longitudinal forces, including the driving force at the rear tyre sufficient to maintain the steady speed. In the second term, $m_{j}$ represents the mass of the $j$ th body, $v_{j}$ is the velocity of the body's mass centre, $w_{j}$ is the body's angular velocity vector and $g$ is the gravitational acceleration vector. Invariably, in a fully established steady turn, $\left|F_{\text {error }}\right|\langle 0.02 \mathrm{~N}$.

In much the same way, the following moment error should be zero:

$$
\underline{M}_{\mathrm{error}}=\sum_{i} \underline{l}_{i} \times \underline{F}_{i}+\sum_{j}\left\{\underline{l}_{j} \times m_{j}\left(\underline{g}^{-} \underline{v}_{j} \times \underline{\omega}_{j}\right)-\underline{\omega}_{j} \times \underline{H}_{j}\right\}+\sum_{k} \underline{M}_{k},
$$


where $\underline{l}_{i}$ and $\underline{l}_{j}$ are moment arm vectors referred to the rear wheel contact point and $\underline{H}_{j}$ is the moment of momentum of body $j$ about its mass centre. The first sum accounts for the moments generated by the external forces listed above, while the second contains a part treating gravitational moments and moments of inertial forces on the body mass centres and a part accounting for the rate of change of moment of momentum of each body, with respect to its mass centre. The third summation deals with aerodynamic pitching and tyre aligning moments.

Each of the terms $\underline{w} \times \underline{H}$ is calculated as $\underline{w} \times\left(H_{x} \underline{i}+H_{y} j+H_{z} \underline{k}\right)$, with $\underline{H}$ having components $H_{x}, H_{y}$ and $H_{z}$ in directions denoted by the unit vectors $\underline{i}, \underline{j}$ and $\underline{k}$, which must be chosen so that the moment of momentum components are invariant, when the motorcycle is in a steady turn. For all the non-spinning bodies, the body reference axes satisfy this requirement. For the wheels, the parent body's reference system needs to be used and a moment of momentum term for the spin added on. For the most general case applicable here in which $I_{x y}$ and $I_{y z}$ are zero but $I_{x z}$ is non-zero [9], noting a change of sign of products of inertia, as compared with the reference, because [9] and Autosim use opposite definitions:

$$
H_{x}=I_{x x} \omega_{x}+I_{x z} \omega_{z} ; \quad H_{y}=I_{y y} \omega_{y} ; \quad \text { and } \quad H_{z}=I_{x z} \omega_{x}+I_{z z} \omega_{z},
$$

in which $\omega_{x}, \omega_{y}$ and $\omega_{z}$ are the components of $\underline{\omega}$ in the $\underline{i}, j$ and $\underline{k}$ directions.

Thus, for the main body, this second component of rate of change of moment of momentum, that about the mass centre, is of the form:

$$
\begin{aligned}
\underline{\omega}_{\text {main }} \times & \left\{\left(I_{\text {main } x} \omega_{x}+I_{\text {main } x z} \omega_{z}\right) \cdot \underline{i}_{\text {main }}+I_{\text {main } y} \omega_{y} \cdot \underline{j}_{\text {main }}\right. \\
& \left.+\left(I_{\text {main } x z} \omega_{x}+I_{\text {main } z} \omega_{z}\right) \cdot \underline{k}_{\text {main }}\right\} .
\end{aligned}
$$

Here, $\underline{i}_{\text {main }}, \underline{j}_{\operatorname{main}}$ and $\underline{k}_{\text {main }}$ denote the unit vectors $\underline{i}, j$ and $\underline{k}$ for the main body. To deal with the rear wheel, its diametral inertia is added to the corresponding terms belonging to the swing arm, its parent body, as if it were part of the swing arm. The spin is accounted for by a term: $\underline{\omega}_{\text {swingarm }} \times I_{r w y} \omega_{y} \cdot[r w y]$ and similarly for the front wheel, for which the lower fork body is the parent. For any steady turn, $\left|M_{\text {error }}\right|<0.02 \mathrm{Nm}$.

The power error is given by:

$$
P_{\text {error }}=\tau \cdot \omega_{\text {spin }}+\sum_{i} \underline{F}_{i} \cdot \underline{v}_{i}+\sum_{k} \underline{M}_{k} \cdot \underline{\omega}_{k},
$$

in which $\tau$ is the rear wheel driving torque and $\omega_{\text {spin }}$ is its spin velocity relative to the swing arm. $\underline{v}_{i}$ is the velocity of the point of application of force, $\underline{F}_{i}$, and $\underline{\omega}_{k}$ is the absolute angular velocity of the body to which moment, $\underline{M}_{k}$, is applied. Describing the power associated with tyre forces requires care also. The velocity involved is that of the tyre tread base material [30], already calculated in connection with finding the slip ratio and the slip angle. For any steady turn, indicating amazing precision, $P_{\text {error }}\langle 0.3 \mathrm{~mW}$. 
These checks on any equilibrium state are substantially independent of the full equations of motion on which the simulation model depends and it is reassuring that they are satisfied.

\section{Typical Results}

The main uses of a model such as that described are (a) general simulation of responses to defined steering control inputs, possibly involving hardware in the loop (b) determination of steady-state equilibrium cornering "trim" states (c) linearization of the equations to represent small motions in the neighbourhood of a trim state (d) root locus calculations for constant lean angle and varying speed or viceversa and (e) frequency response calculations to find gains and phases in sustained motion involving sinusoidal forcing from the steering system or from road undulations [30]. The power computations also allow determination in detail of where the engine power is dissipated in steady turning.

Trim state determination is a necessary forerunner to stability and frequency response computations, to enable the linearisation to be done correctly. Also, as described in Section 6, speed and lean angle controllers are likely to be needed to allow the trim states to be found, over a reasonably full range of feasible speeds and lean angles. Some of these uses and some behavioural properties of the machine in focus are illustrated next.

The model was first used to simulate a straight line run from 1 to $75 \mathrm{~m} / \mathrm{s}$ with a very small constant acceleration of $0.05 \mathrm{~m} / \mathrm{s}^{2}$. This gives the trim state, changing with the speed, from which small perturbations are considered to occur and for which a linearised model is appropriate. The linearised model, having a free steering system with the feedback steering controller disabled, was then used to obtain the root locus plot shown in Figure 18. The machine, as represented, is stable for straight running throughout the speed range above about $6 \mathrm{~m} / \mathrm{s}$. Also shown in Figure 18 are the loci for the nominal motorcycle but with the rear frame torsional stiffness divided by 2 and then 4 with the frame twist damping coefficient reduced by factors of 0.7071 and 0.5 respectively.

The high speed weave stability is compromised significantly by the reduction in stiffness and the wobble problem is transferred from high speed to medium speed by these changes. This aligns with earlier findings, that flexible frames promote medium speed wobble, while very stiff frames give more of a potential problem at high speeds, implying the need for a steering damper to ensure adequate margins.

The damping coefficient associated with the rider upper body lean freedom is now varied, with root loci being shown in Figure 19. Rider damping can be seen to influence the weave mode only where the damping is plentiful but it contributes usefully to the stability of the wobble mode at high speed. The results are consistent with the idea that lighter riders are more likely to suffer wobble oscillations than heavier ones, in accord with anecdotal evidence. 


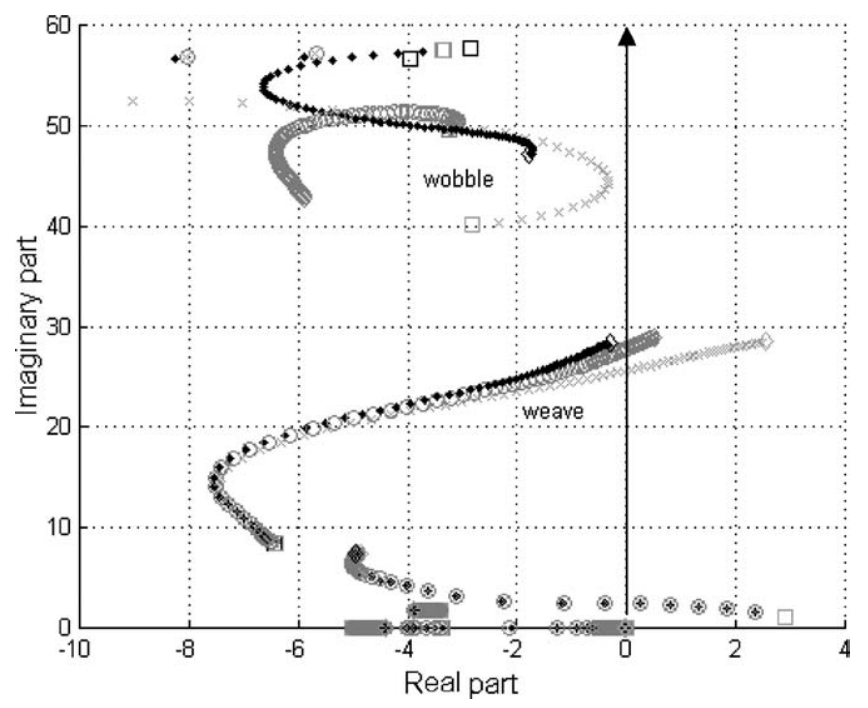

Figure 18. Root locus plot for straight running through speed range 1.1 (squares) to 75 (diamonds) $\mathrm{m} / \mathrm{s}$. Nominal machine, points; frame stiffness halved, circles; frame stiffness quartered, crosses (with damping adjustments).

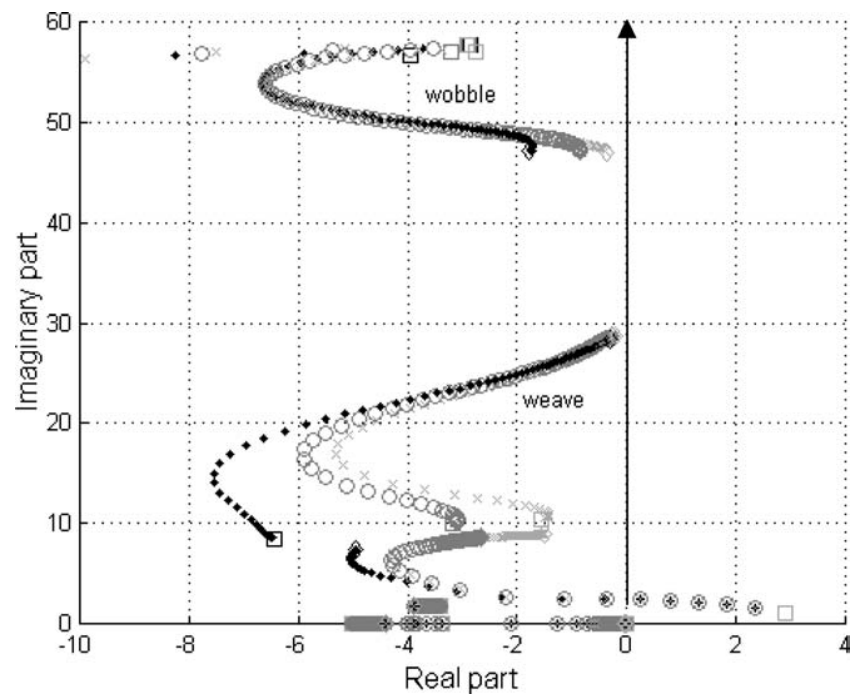

Figure 19. Root locus plot for straight running through 1.1 (squares) to 75 (diamonds) $\mathrm{m} / \mathrm{s}$ speed for nominal machine (points) and with the rider lean damping coefficient factored by 0.5 (circles) and 0.25 (crosses).

The behaviour of the motorcycle in quasi-steady turning at a sustained lean angle of $30^{\circ}$ with a small forward acceleration of $0.05 \mathrm{~m} / \mathrm{s}^{2}$ is illustrated in Figure 20 . The steer angle is small, except at low speeds, for which it rises markedly. It changes sign at just over $20 \mathrm{~m} / \mathrm{s}$, where the fixed control motorcycle becomes self-stabilising [1]. 

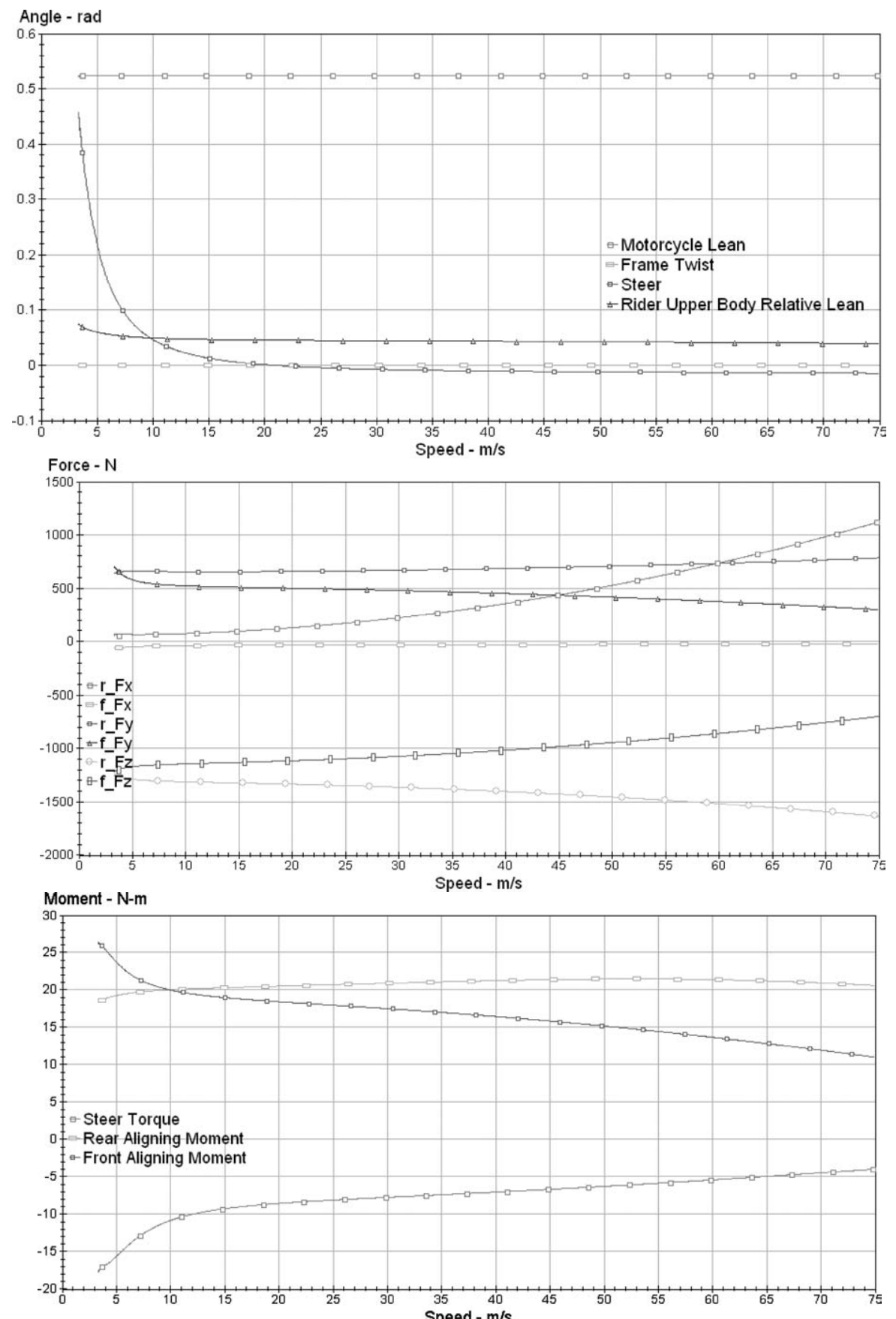

Figure 20. (a) State variables (b) tyre forces (c) steering torque and tyre aligning moments in a sustained $30^{\circ}$ lean angle turn accelerating at $0.05 \mathrm{~m} / \mathrm{s}^{2}$ from 3.7 to $75 \mathrm{~m} / \mathrm{s}$. 
The frame twist is imperceptibly small, despite its importance to the dynamics. The rider leans into the turn to a moderate degree. The tyre loads and shear forces are shown, indicating that the rear tyre will be near to its friction limits at the top end of the speed range covered. The steer torque required can be seen to be somewhat less than the front tyre aligning moment, all through the speed range. Predicting the steering torque accurately apparently depends on modelling the front tyre aligning moment well. Close inspection of the motorcycle lean angle record shows that there is some interaction between the lean angle controller and the acceleration. The lean angle is not maintained precisely on target and the greater the lean angle and the acceleration are, the larger the errors become. The simplest solution is to use very low acceleration levels but the simulation runs then take a long time to complete. If an unstable condition occurs during a run, that run is lost, so that long runs are potentially problematic.

An alternative procedure has been developed. This involves describing the speed target by a saturating ramp, so that true equilibrium is established at the finish of a run. A typical run will increment the speed by $5 \mathrm{~m} / \mathrm{s}$ only (less at low speed) and the final state of one run is used as the initial state for the following one. A whole series of runs constitutes the equilibrium data for one lean angle. From such a series for $45^{\circ}$ lean, contributions to the power dissipated, as functions of speed, are shown in Figure 21. It will be no surprise to see that most of the engine power is used to overcome aerodynamic drag, especially at high speeds, but it is not at all obvious that each tyre's aligning moment may dissipate $4 \mathrm{~kW}$ at high speed. Also, at high speed, the rear tyre driving force accounts for a relatively high power dissipation through longitudinal slipping.

Using a quasi-steady run at $15^{\circ}$ lean, then the results above for $30^{\circ}$ and again corresponding results for $45^{\circ}$ for the trim state data used in the linearization, the root loci for the nominal machine are shown in Figures 22-24. Each figure also contains similar results, similarly obtained, for the motorcycle with half the frame torsional stiffness and 0.7071 times the frame damping coefficient. Damping of the oscillatory modes improves with cornering except that the medium speed wobble damping at $45^{\circ}$ lean becomes quite small. In each case, it is clear that halving the frame stiffness is detrimental to the stability properties. The more elaborate procedure for establishing steady-state equilibria yields root loci which are indistinguishable from those shown; that is, the influence of the small acceleration employed is negligible in these cases.

If the cornering motorcycle is excited by regular road undulations, the response is potentially dangerous if resonance in connection with a lightly damped mode of oscillation occurs [30]. It was found in earlier work that about $15^{\circ}$ lean is likely to represent a worst case, since, for smaller angles, the road forcing couples only weakly to the lateral oscillatory responses, while for larger angles, the modal damping is likely to increase. Such a $15^{\circ}$ lean case is illustrated, for a constant speed of $65 \mathrm{~m} / \mathrm{s}$, in Figure 25. The plot shows the steer angle to road displacement forcing frequency response gain relative to $1 \mathrm{rad} / \mathrm{m}$, accounting properly 

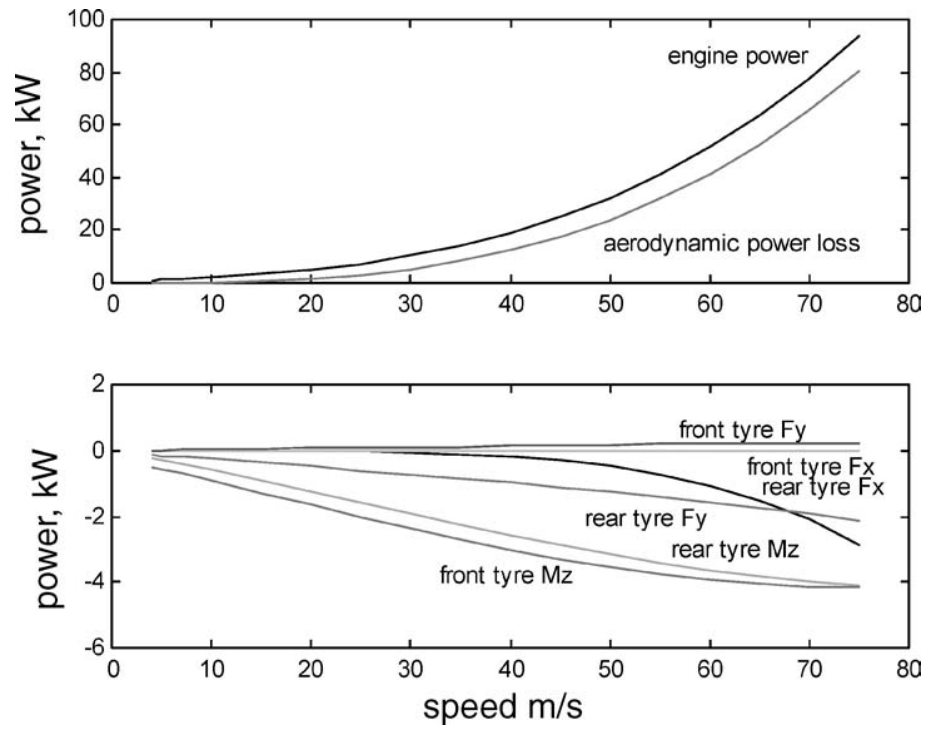

Figure 21. Power contributions as functions of speed in steady turning with $45^{\circ}$ lean angle.

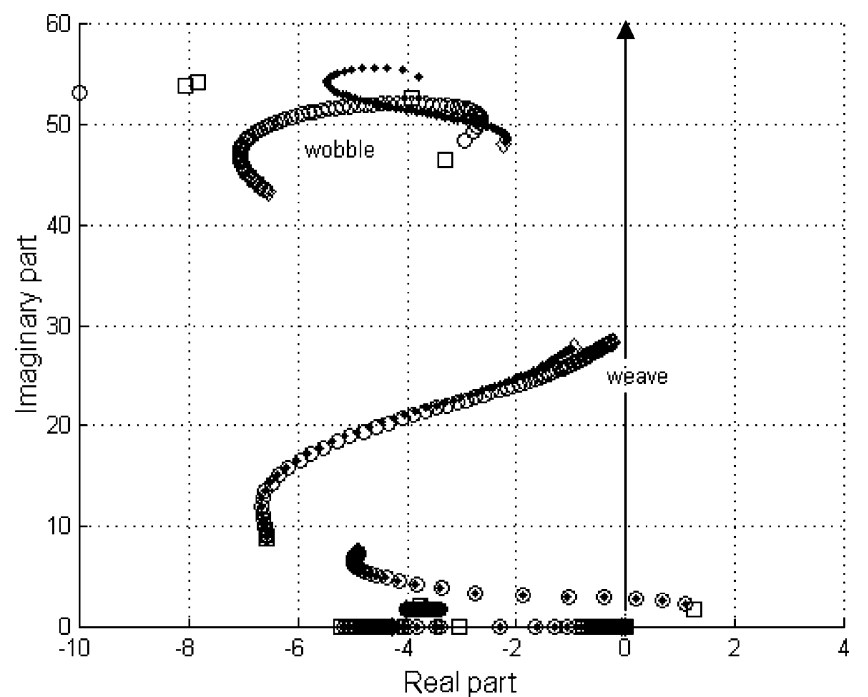

Figure 22. Motorcycle root locus plot for $15^{\circ}$ lean angle through speed range 3.3 (squares) to 75 (diamonds) m/s. Nominal case - points; frame stiffness halved - circles.

for the time delay between the forcing acting on the front wheel and on the rear wheel, the so-called wheelbase filtering effect. Resonance of the cornering weave is evident at $26 \mathrm{rad} / \mathrm{s}$ forcing frequency, while the wobble is most responsive at $50 \mathrm{rad} / \mathrm{s}$. 


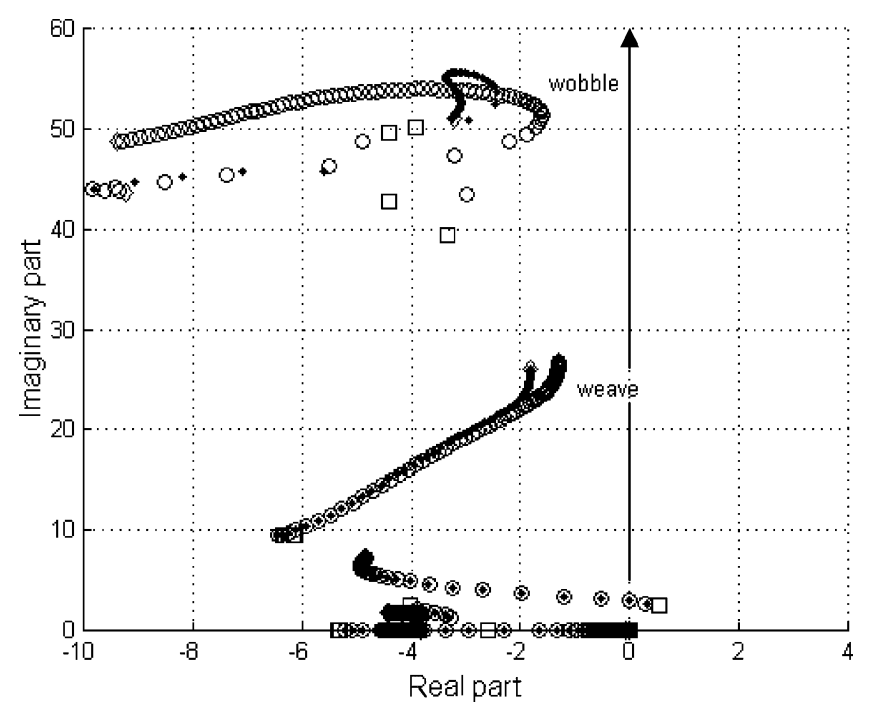

Figure 23. Motorcycle root locus plot for $30^{\circ}$ lean angle through speed range 3.8 (squares) to 75 (diamonds) m/s. Nominal case - points; frame stiffness halved - circles.

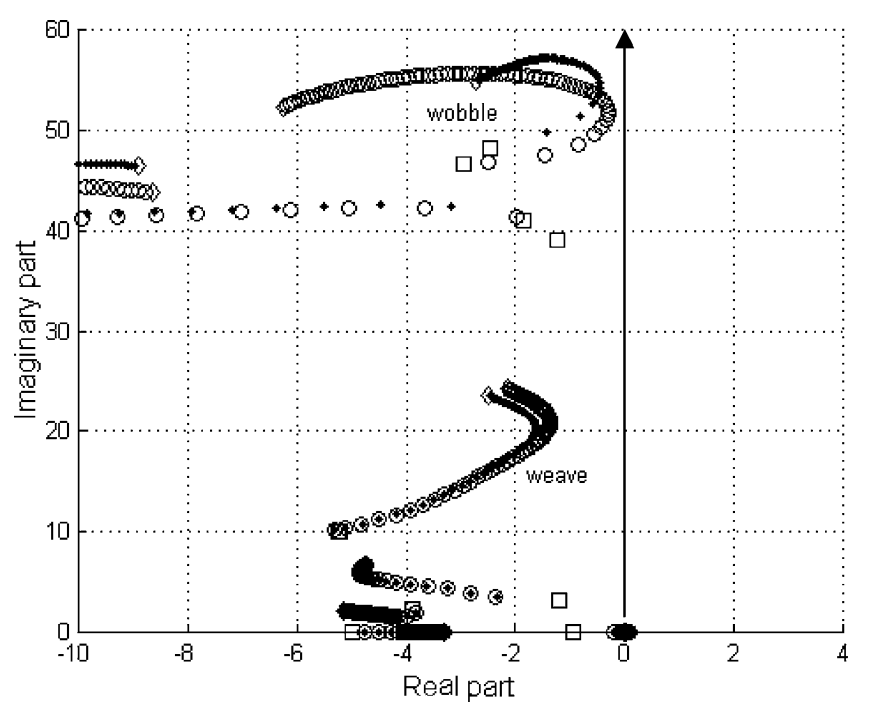

Figure 24. Motorcycle root locus plot for $45^{\circ}$ lean angle through speed range 5.8 (squares) to 75 (diamonds) $\mathrm{m} / \mathrm{s}$. Nominal case - points; frame stiffness halved - circles.

\section{Conclusions}

Substantial improvements to an advanced motorcycle dynamics model have been made, relating to (a) tyre/road contact geometry; (b) the tyre shear force and moment system; (c) tyre relaxation properties and (d) the monoshock rear suspension 


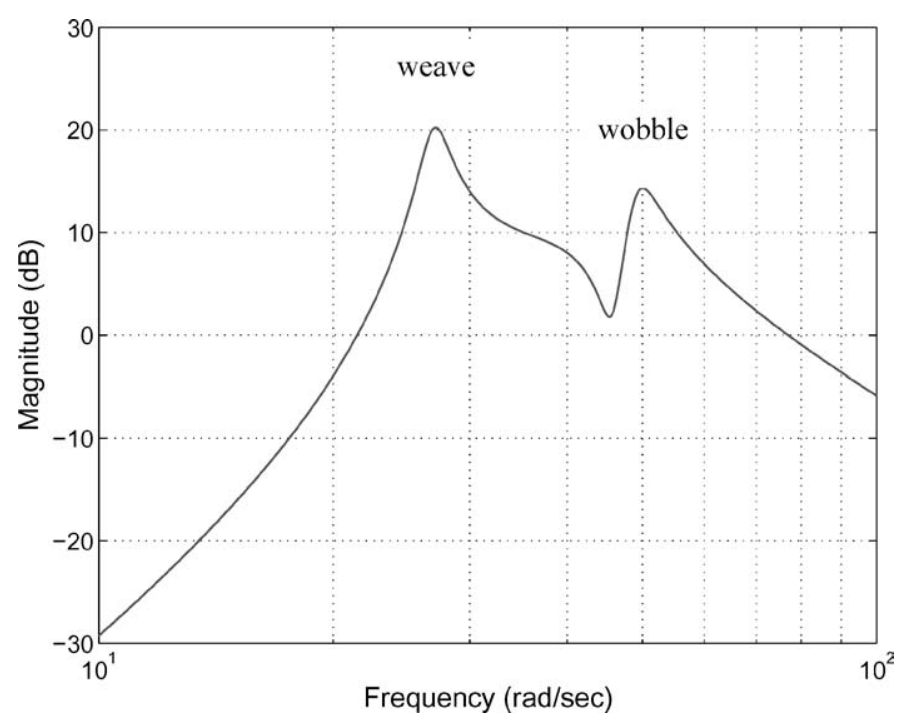

Figure 25. Steer angle response to road undulation forcing of nominal motorcycle at $15^{\circ}$ lean angle and $65 \mathrm{~m} / \mathrm{s}$ speed.

mechanism. In particular, parameters for the powerful Magic Formula method, representing the shear forces developed by modern, high performance motorcycle tyres have been derived. This provides a readily usable generic description of the steady-state force and moment system of such tyres, with a very wide range of validity. Also, the geometric treatment of the monoshock suspension system is new and it contributes to computational efficiency. Steady-turning equilibrium force, moment and power checks have been refined and results of high precision shown testify to the model's accuracy of construction.

Significant progress towards a complete parametric description of a contemporary, high performance motorcycle has been made, although a little further work is needed to finish the measurement campaign. The rider upper body structure has been represented as relatively compliant, in sympathy with the rig measurements of Nishimi et al [16]. Results obtained on this basis suggest that the rider upper body damping is significantly stabilising to the wobble mode, accounting potentially for the observation that light riders are more at risk from oscillations than heavier ones.

Steady turning equilibrium states, tyre forces and steer torque requirements have been illustrated and the power dissipation through the speed range for steady turning at $45^{\circ}$ lean angle has been shown for the first time.

Straight running root locus plots, from a linearised version of the model, have suggested that, despite the relatively high torsional stiffness of many modern frames, it is still important to the stability and control and it needs including in analysis and design discussions. Use of the model for the calculation of stability in cornering has been illustrated. Stability margins in cornering typically increase as compared with straight running, although complex patterns of behaviour are possible. Current work 
concerns the nonlinear phenomena, sub-harmonic and super-harmonic oscillations, special operating conditions yielding commensurate relationships between natural frequencies and the consequent possibility of internal and combination resonances, and advantageous alternatives to the conventional steering damper for restraining the steering system.

\section{Appendix: Motorcycle Parameter Values (SI Units)}

Table A.I. Masses.

\begin{tabular}{lllllll}
\hline Mff_str & Mff_sus & Mmain & Mrw & Mfw & Mubr & Mswg_arm \\
9.99 & 7.25 & 165.13 & 14.7 & 11.9 & 33.68 & 8 \\
\hline
\end{tabular}

Table A.II. Inertias.

\begin{tabular}{llllllllll}
\hline Iff_strx & Iff_stry & Iff_strz & Iff_strxz & Imnx & Imny & Imnz & Imnxz & Iubrx & Iubry \\
1.341 & \multirow{2}{*}{1.584} & 0.4125 & 0 & 11.085 & 22.013 & 14.982 & -3.691 & 1.428 & 1.347 \\
\hline \multirow{2}{*}{ Iubrz } & \multirow{2}{*}{ Iubrxz } & \multirow{2}{*}{ Ifwx } & \multirow{2}{*}{ Ifwy } & \multirow{2}{*}{ Irwx } & Irwy & Is_ax & Is_ay & Is_az & \\
0.916 & 0.443 & 0.270 & 0.484 & 0.383 & 0.638 & 0.02 & 0.259 & 0.259 & \\
\hline
\end{tabular}

Table A.III. Dimensions (Figures 1, 3, 4 and 16).

\begin{tabular}{|c|c|c|c|c|c|c|c|c|}
\hline $\mathrm{x} 2$ & $z 2$ & $x 3$ & $z 3$ & $x 4$ & $z 4$ & $x 5$ & $z 5$ & $x 6$ \\
\hline 1.173 & -0.749 & 1.164 & -0.77 & 1.342 & -0.426 & 1.365 & -0.324 & 1.410 \\
\hline z6 & $\mathrm{z} 7$ & $\mathrm{x} 8$ & z8 & $\mathrm{x} 9$ & z9 & $\mathrm{x} 10$ & $\mathrm{z} 10$ & $\mathrm{x} 11$ \\
\hline-0.282 & -0.297 & 0.6779 & -0.4724 & 0.364 & -0.8438 & 0.415 & -1.14 & 0.549 \\
\hline $\mathrm{z} 11$ & $\mathrm{x} 13$ & z13 & x14 & $\mathrm{z} 14$ & x19 & z19 & $\mathrm{x} 20$ & $\mathrm{z} 20$ \\
\hline-0.3608 & 0.487 & -0.4888 & 0.196 & -0.3113 & 0.539 & -0.1878 & 0.4946 & -0.1522 \\
\hline $\mathrm{x} 21$ & $\mathrm{z} 21$ & $x 22$ & $\mathrm{z} 22$ & $\varepsilon$ & r_R0 & f_R0 & 1_free & \\
\hline 0.4443 & -0.1782 & 0.3722 & -0.2748 & 0.4189 & 0.095 & 0.06 & 0.3435 & \\
\hline
\end{tabular}

Table A.IV. Limit stop geometry.

\begin{tabular}{lllll}
\hline r_lmax & r_lmin & f_dmax & f_dmin & str_lim \\
0.3385 & 0.2735 & 0.03 & 0.07 & 0.5061 \\
\hline
\end{tabular}

Table A.V. Stiffnesses.

\begin{tabular}{llllll}
\hline r_k & f_k & r_kt & f_kt & r_krbd & f_krbd \\
58570 & 25000 & 141000 & 130000 & $1 \mathrm{e} 15$ & $1 \mathrm{e} 15$ \\
r_kcom & f_kcom & kp_ubr & kp_twst & kp_str & k_strstop \\
$1 \mathrm{e} 11$ & $1 \mathrm{e} 11$ & 380 & 100000 & 0 & $3 \mathrm{e} 9$ \\
\hline
\end{tabular}


Table A.VI. Damping coefficients.

\begin{tabular}{lllll}
\hline r_c & f_c & Cp_ubr & Cp_twst & Cp_str \\
11650 & 2134 & 34.0 & 100 & 6.944 \\
\hline
\end{tabular}

Table A.VII. Aerodynamic parameters.

\begin{tabular}{lllll}
\hline $\mathrm{CD}$ & $\mathrm{CL}$ & $\mathrm{CP}$ & $\mathrm{f}_{-}$Area & $\rho$, density \\
0.48 & 0.078 & 0.189 & 0.65 & 1.225 \\
\hline
\end{tabular}

Table A.VIII. Speed and steering control gain coefficients.

\begin{tabular}{llllllll}
\hline drvp & drvi & $\operatorname{spg} 0$ & $\operatorname{spg} 1$ & sig0 & sig1 & $\operatorname{sdg} 0$ & $\operatorname{sdg} 1$ \\
-500 & -1000 & -60 & -0.6875 & -250 & 1.875 & -50 & 0.6 \\
\hline
\end{tabular}

\section{References}

1. Sharp, R.S., 'Stability, control and steering responses of motorcycles', Vehicle System Dynamics 35(4-5), 2001, 291-318.

2. Koenen, C., 'The dynamic behaviour of a motorcycle when running straight ahead and when cornering', Doctoral Dissertation, Delft University, 1983.

3. Wisselman, D., Iffelsberger, D. and Brandlhuber, B., 'Einsatz eines FahrdynamikSimulationsmodells in der Motorradentwicklung bei BMW', ATZ 95(2), 1993.

4. Breuer, T. and Pruckner, A., 'Advanced dynamic motorbike analysis and driver simulation', 13th European ADAMS Users' Conference, Paris, 1998.

5. Sharp, R.S. and Limebeer, D.J.N., 'A motorcycle model for stability and control analysis', Multibody System Dynamics 6(2), 2001, 123-142.

6. Berritta, R., Biral, F. and Garbin, S., 'Evaluation of motorcycle handling by multibody modeling and simulation', Proc. 6th Int. Conf. on High Tech. Engines and Cars, Modena, 2000.

7. Cossalter, V. and Lot, R., 'A motorcycle multi-body model for real time simulations based on the natural coordinates approach', Vehicle System Dynamics 37(6), 423-447, 2002.

8. Bio-Astronautus Data Book, NASA SP 3006, 1964.

9. Housner, G.W. and Hudson, D.E., Applied Mechanics-Dynamics, 2nd edn., D. Van Nostrand Company Inc., Princeton, NJ, 1959.

10. Pick, A. and Cole, D.J., 'Neuromuscular dynamics and the vehicle steering task', in Proc. IAVSD Symposium on Dynamics of Vehicles on Roads and on Tracks, Kanagawa, Japan, August 2003, in press.

11. Sharp, R.S. and Limebeer, D.J.N., 'On steering wobble oscillations of motorcycles', Proc. I. Mech. E., Part C, Journal of Mechanical Engineering Science, in press.

12. Abdelkebir, A., 'Measurements of inertia, stiffness and damping components of a Suzuki GSXR1000', Project work report, Imperial College London, Mechanical Engineering Department, 2002.

13. Sharp, R.S. and Alstead, C.J., 'The influence of structural flexibilities on the straight running stability of motorcycles', Vehicle System Dynamics 9(6), 1980, 327-357.

14. Spierings, P.T.J., 'The effects of lateral front fork flexibility on the vibrational modes of straightrunning single track vehicles', Vehicle System Dynamics 10(1), 1981, 37-38. 
15. Giles, C.G. and Sharp, R.S., 'Static and dynamic stiffness and deflection mode measurements on a motorcycle, with particular reference to steering behaviour', Proc. I. Mech. E./MIRA Conference on Road Vehicle Handling, London, Mechanical Engineering Publications, 1983, 185-192.

16. Nishimi, T., Aoki, A. and Katayama, T., 'Analysis of straight running stability of motorcycles', 10th International Technical Conf. on Experimental Safety Vehicles, July 1985, 33 pp.

17. Cossalter, V., Doria, A. and Lot, R., 'Steady turning of two-wheeled vehicles', Vehicle System Dynamics 31(3), 1999, 157-181.

18. Cossalter, V., Lot, R. and Fabiano, M., 'The influence of tire properties on the stability of a motorcycle in straight running and curves', SAE 2002-01-1572, 2002.

19. Cossalter, V., Motorcycle Dynamics, Race Dynamics, Greendale, WI, 2002.

20. de Vries, E.J.H. and Pacejka, H.B., 'Motorcycle tyre measurements and models', in Palkovics, L. (ed.), Proceedings of the 15th IAVSD Symposium on the Dynamics of Vehicles on Roads and on Tracks, 1997 (Suppl. Vehicle System Dynamics) 28, 1998.

21. de Vries, E.J.H. and Pacejka, H.B., 'The effect of tyre modeling on the stability analysis of a motorcycle', Proceedings of AVEC'98, Nagoya, SAE of Japan, 1998, 355-360.

22. Tezuka, Y., Ishii, H. and Kiyota, S., 'Application of the magic formula tire model to motorcycle maneuverability analysis', JSAE Review 22, 2001, 305-310.

23. Pacejka, H.B., Tyre and Vehicle Dynamics, Butterworth Heinemann, Oxford, 2002.

24. Sakai, H., Kanaya, O. and Iijima, H., 'Effect of main factors on dynamic properties of motorcycle tires', SAE 790259, 1979.

25. Cossalter, V., Doria, A., Lot, R., Ruffo, N. and Salvador, M., 'Dynamic properties of motorcycle and scooter tires: Measurement and comparison', Vehicle System Dynamics 39(5), 2003, 329_ 352.

26. van Oosten, J.J.M., Kuiper, E., Leister, G., Bode, D., Schindler, H., Tischleder, J. and Köhne, S., 'A new tyre model for TIME measurement data', in press.

27. Fujioka, T. and Goda, K., 'Discrete brush tire model for calculating tire forces with large camber angle', in Segel, L. (ed.), Proceedings of the 14th IAVSD Symposium on the Dynamics of Vehicles on Roads and on Tracks, 1995 (Suppl. Vehicle System Dynamics) 25, 1996.

28. Ishii, H. and Tezuka, Y., 'Considerations of turning performance for motorcycles', in Proceedings of SETC'97, Yokohama, 1997, 383-389 (JSAE 9734601; SAE 972127).

29. Mousseau, C.W., Sayers, M.W. and Fagan, D.J., 'Symbolic quasi-static and dynamic analyses of complex automobile models', The Dynamics of Vehicles on Roads and on Tracks, Sauvage, G. (ed.), in Proceedings of the 12th IAVSD Symposium, Swets and Zeitlinger, Lisse, 1992, 446-459.

30. Limebeer, D.J.N., Sharp, R.S. and Evangelou, S., 'Motorcycle steering oscillations due to road profiling', Proc. ASME, Journal of Applied Mechanics 69(6), 2002, 724-739. 